\title{
Assembling Polycyclic Bisguanidine Motifs Resembling Batzelladine Alkaloids by Double Tethered Biginelli Condensations
}

\author{
Frederick Cohen, Shawn K. Collins and Larry E. Overman * \\ Department of Chemistry, 516 Rowland Hall, University of California, Irvine, CA 92697-2025
}

\section{Supporting Information:}

Experimental procedures and characterization data for bis- $\square$-ketoesters 6, 10-15; double Biginelli products 9, 16-21, 25 and 27; copies of ${ }^{1} \mathrm{H}$ and ${ }^{13} \mathrm{C}$ spectra for these new compounds, selected minor double Biginelli products and $23\left(\mathrm{R}=n-\mathrm{C}_{7} \mathrm{H}_{15}\right)$ (33 pages).

General Experimental. All reactions were carried out under a $\mathrm{N}_{2}$ or Ar atmosphere in oven-dried and base-washed glassware. Tetrahydrofuran (THF), diethyl ether, and dichloromethane were degassed with argon and then passed through two 4 x 36 inch columns of anhydrous neutral A-2 alumina (8 x 14 mesh; activated under a flow of $\mathrm{Ar}$ at $350{ }^{\circ} \mathrm{C}$ for $3 \mathrm{~h}$ ) to remove water. ${ }^{1}$ Toluene (PhMe) was degassed with argon, passed through one 4 × 36 inch column of Q-5 reactant (activated under a flow of 5\% hydrogen/nitrogen at $250{ }^{\circ} \mathrm{C}$ for $3 \mathrm{~h}$ ) to remove oxygen and then through one $4 \times 36$ inch column of anhydrous alumina to remove water. ${ }^{1}$ Silica gel (0.040-0.063 mesh) was used for flash chromatography unless otherwise stated. Molarities of organolithium reagents were established by titration with diphenylacetic acid. Trifluoroethanol (TFE) was purchased from Aldrich and used without further purification. ${ }^{1} \mathrm{H}$ NMR chemical shifts are reported as $\square$ values in ppm relative to $\mathrm{CHCl}_{3}$ or $\mathrm{MeOH}$. ${ }^{1} \mathrm{H}$ NMR coupling constants are reported in $\mathrm{Hz}$ and refer to apparent multiplicities and not true coupling constants. Multiplicity is indicated as follows: s (singlet); d (doublet); q (quartet); m (multiplet); app d (apparent doublet); app t (apparent triplet); dd (doublet of doublets); ddd (doublet, doublet of doublets); dddd (doublet, doublet, doublet of doublets); br s (broad singlet). ${ }^{1} \mathrm{H}$ NMR spectra of guanidine double Biginelli products show few resolved signals; these spectra are not tabulated, but the original spectra are reproduced. The IR spectra absorptions are recorded as wavenumbers $\left(\mathrm{cm}^{-1}\right)$.

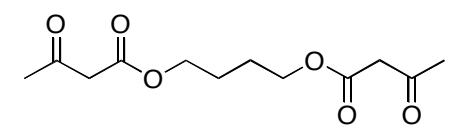

3-Oxobutyric acid 4-(3-oxobutyryloxy)butyl ester (10). General procedure for preparing bis- $\square$-ketoesters using the Taber protocol. ${ }^{2}$ A solution of DMAP (677 mg, 5.55 mmol), methyl

\footnotetext{
${ }^{1}$ Pangborn, A. B.; Giardello, M. A.; Grubbs, R. H.; Rosen, R. K.; Timmers, F. J. Organometallics 1996, $15,1518$.

2 Taber, D. F.; Amedio, J.C.; Patel, Y. K. J. Org. Chem. 1985, 50, 3618.
} 
acetoacetate $(1.8 \mathrm{~mL}, 17 \mathrm{mmol}), 1,4-$ butanediol $(500 \mathrm{mg}, 5.5 \mathrm{mmol})$ and toluene $(10 \mathrm{~mL})$ was heated to 110 ${ }^{\circ} \mathrm{C}$ for $18 \mathrm{~h}$. The reaction was allowed to cool to room temperature and then was concentrated in vacuo. The residue was purified by chromatography (1:4 ethyl acetate:hexanes) to afford $1.29 \mathrm{~g}(90 \%)$ of $\mathbf{1 0}$ as a yellow oil: ${ }^{1} \mathrm{H}$ NMR (500 MHz, $\mathrm{CDCl}_{3}$ ) $\square 4.04-4.02(\mathrm{~m}, 4 \mathrm{H}), 3.36(\mathrm{~s}, 4 \mathrm{H}), 2.13$ (s, 6H), 1.62-1.59 (m, $4 \mathrm{H}) ;{ }^{13} \mathrm{C}$ NMR $\left(125 \mathrm{MHz}, \mathrm{CDCl}_{3}\right.$ ) $\square 200.25,166.6,64.2,49.42,29.65,24.6$; IR (thin film) 2964, 1737, $1713,1648,1412,1316,1175,1038,955 \mathrm{~cm}^{-1}$; HRMS $\left(\mathrm{CI}^{+}\right) \mathrm{m} / z .259 .1180$ (259.1181 calcd for $\mathrm{C}_{12} \mathrm{H}_{19} \mathrm{O}_{6}$ $\left.[\mathrm{M}+\mathrm{H}]^{+}\right)$.

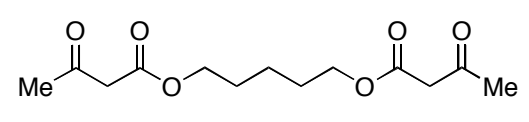

3-Oxododecanoic acid 4-(3-oxododecanoyloxy)butyl ester (6). Purification by chromatography (5:1-1:1 hexanes:EtOAc) afforded 85\% of 6 as a colorless oil: ${ }^{1} \mathrm{H}$ NMR (400 MHz, $\left.\mathrm{CDCl}_{3}\right) \square 4.2-4.1(\mathrm{~m} \mathrm{4H}), 3.46(\mathrm{~s}, 4 \mathrm{H}), 2.27(\mathrm{~s}, 6 \mathrm{H}), 1.72-1.65(\mathrm{~m}, 4 \mathrm{H}), 1.47-1.41(\mathrm{~m}, 2 \mathrm{H}) ;{ }^{13} \mathrm{C} \mathrm{NMR}$ $\left(100 \mathrm{MHz}, \mathrm{CDCl}_{3}\right) \square 200.5,167.1,65.0,50.0,30.2,28.0,22.2$; IR (thin film) 2957, 1741, 1717, 1319, $1152 \mathrm{~cm}^{-1}$; HRMS $\left(\mathrm{CI}^{+}\right) \mathrm{m} / z 273.1339$ (273.1340 calcd for $\mathrm{C}_{13} \mathrm{H}_{21} \mathrm{O}_{6}, \mathrm{MH}^{+}$).

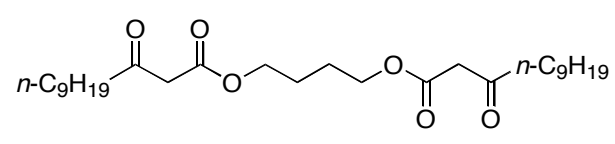

3-Oxododecanoic acid 4-(3-oxododecanoyloxy)butyl ester (11). Purification by chromatography (10:1 hexanes:EtOAc) afforded $48 \%$ of $\mathbf{1 1}$ as a colorless oil: ${ }^{1} \mathrm{H}$ NMR (400 MHz, $\left.\mathrm{CDCl}_{3}\right) \square 4.20-4.18(\mathrm{~m}, 4 \mathrm{H}), 3.43(\mathrm{~s}, 4 \mathrm{H}), 2.52(\mathrm{dt}, J=10.0,1.8 \mathrm{~Hz}, 4 \mathrm{H}), 1.71-1.63(\mathrm{~m}, 2 \mathrm{H}), 1.61-1.56$ $(\mathrm{m}, 4 \mathrm{H}), 1.45-1.39(\mathrm{~m}, 2 \mathrm{H}), 1.30-1.18(\mathrm{~m}, 24 \mathrm{H}), 0.90(\mathrm{t}, J=8.0 \mathrm{~Hz}, 6 \mathrm{H}) ;{ }^{13} \mathrm{C} \mathrm{NMR}\left(125 \mathrm{MHz}, \mathrm{CDCl}_{3}\right) \square$ 202.6, 167.06, 64.4, 48.9, 42.9, 31.7, 29.25, 29.2, 29.1, 28.9, 23.3, 22.5, 13.9; IR (thin film) 2920, 1738, 1413, 1321, 1267, 1159, 1050, 965, $733 \mathrm{~cm}^{-1}$; HRMS $\left(\mathrm{CI}^{+}\right) \mathrm{m} / z$. 483.3686 (483.3685 calcd for $\mathrm{C}_{29} \mathrm{H}_{50} \mathrm{O}_{6}$, $\left.\mathrm{MH}^{+}\right)$.

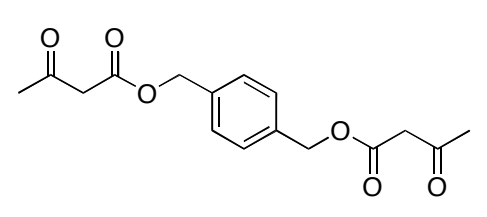

3-Oxobutyric acid 4-(3-oxobutyryloxymethyl)benzyl ester (12). Purification by chromatography (5:1 hexanes:EtOAc) afforded $70 \%$ of $\mathbf{1 2}$ as a colorless powder: ${ }^{1} \mathrm{H}$ NMR (500 MHz, $\left.\mathrm{CDCl}_{3}\right) \square 7.42(\mathrm{~s}, 4 \mathrm{H}), 5.23(\mathrm{~s}, 4 \mathrm{H}), 3.56(\mathrm{~s}, 4 \mathrm{H}), 2.31(\mathrm{~s}, 6 \mathrm{H}) ;{ }^{13} \mathrm{C}\left(125 \mathrm{MHz}, \mathrm{CDCl}_{3},\right) \square 200.5,167.1$, 135.8, 128.8, 66.9, 50.21, 30.4; IR (thin film) 2960, 1742, 1713, 1410, 1316, 1268, 1148, 1032, $808 \mathrm{~cm}^{-1}$; HRMS $\left(\mathrm{CI}^{+}\right) \mathrm{m} / z$ 324.1447 (324.1447 calcd for $\mathrm{C}_{16} \mathrm{H}_{18} \mathrm{O}_{6},\left[\mathrm{M}+\mathrm{NH}_{4}\right]^{+}$). 


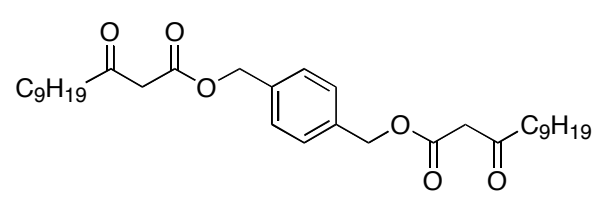

3-Oxododecanoic acid 4-(3-oxododecanoyloxymethyl)benzyl ester (13). Purification by chromatography (4:1 hexanes:EtOAc) afforded $61 \%$ of $\mathbf{1 3}$ as a colorless oil that solidifies at $0{ }^{\circ} \mathrm{C}:{ }^{1} \mathrm{H}$ NMR (500 MHz, $\left.\mathrm{CDCl}_{3}\right) \square 7.35(\mathrm{~s}, 4 \mathrm{H}), 5.16(\mathrm{~s}, 4 \mathrm{H}), 3.47(\mathrm{~s}, 4 \mathrm{H}), 2.50(\mathrm{t}, J=7.3 \mathrm{~Hz}, 4 \mathrm{H}), 1.58-1.56(\mathrm{~m}$, $4 \mathrm{H}), 1.28-1.24(\mathrm{~m}, 24 \mathrm{H}), 0.87(\mathrm{t}, J=5.8 \mathrm{~Hz}, 6 \mathrm{H}) ;{ }^{13} \mathrm{C} \mathrm{NMR}\left(125 \mathrm{MHz}, \mathrm{CDCl}_{3}\right) \square 202.6,167.0,135.5$, 128.4, 88.6, 66.5, 49.1, 43.1, 31.8, 29.3, 29.3, 29.2, 28.9, 23.3, 22.6, 14.0; IR (thin film) 2927, 2858, 1746, 1406, 1220, $911 \mathrm{~cm}^{-1}$; HRMS (ESI) $m / z 553.3497$ (553.3505 calcd for $\mathrm{C}_{32} \mathrm{H}_{50} \mathrm{O}_{6},[\mathrm{M}+\mathrm{Na}]^{+}$).

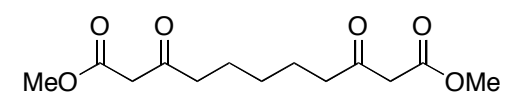

3,9-Dioxoundecanedioic acid dimethyl ester (14). Following the general procedure of Weiler, ${ }^{3}$ $\mathrm{NaH}$ (413 mg, 60\% dispersion, $10.4 \mathrm{mmol})$ was added slowly to a solution of methyl acetoacetate $(1.0 \mathrm{~g}$, $8.62 \mathrm{mmol})$ and THF $(15 \mathrm{~mL})$ at $0{ }^{\circ} \mathrm{C}$. The opaque reaction mixture was stirred for $10 \mathrm{~min}$ at $0{ }^{\circ} \mathrm{C}$, then $n$ BuLi (3.1 mL, $9.5 \mathrm{mmol}$ ) was added dropwise. The resulting yellow reaction mixture was stirred for an additional 10 minutes at $0{ }^{\circ} \mathrm{C}$ before 1,3-diiodopropane (500 uL, $\left.4.3 \mathrm{mmol}\right)$ was added dropwise. The reaction mixture was allowed to warm to room temperature over $30 \mathrm{~min}$ and then was quenched by the addition of $0.1 \mathrm{~N} \mathrm{HCl}(\sim 2 \mathrm{~mL})$. Silica gel was added, the reaction mixture was concentrated and the residue purified by chromatography (4:1 hexanes:EtOAc) to afford $1.82 \mathrm{~g}$ (77\%) of $\mathbf{1 4}$ as a pale yellow liquid: ${ }^{1} \mathrm{H}$ NMR (400 MHz, $\mathrm{CDCl}_{3}$ ) $\square 3.48(\mathrm{~s}, 6 \mathrm{H}), 3.24(\mathrm{~s}, 4 \mathrm{H}), 2.33$ (t, J = 7.1 Hz, 4H), 1.39-1.32 (m, 4H), 1.11-1.04 (m, 4H); ${ }^{13} \mathrm{C}$ NMR (100 MHz, $\left.\mathrm{CDCl}_{3}\right) \square 202.1,167.1,51.5,48.3$. 41.9, 27.6, 22.4; IR (thin film) 2954, 2923, 2854, 1744, 1717, 1457, 1320, 1156, $908 \mathrm{~cm}^{-1}$; HRMS $\left(\mathrm{CI}^{+}\right) \mathrm{m} / z 273.1333$ (273.1338 calcd for $\mathrm{C}_{13} \mathrm{H}_{21} \mathrm{O}_{6}, \mathrm{MH}^{+}$).

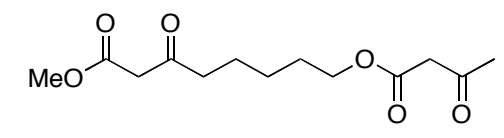

3-Oxo-8-(3-oxobutyryloxy)octanoic acid methyl ester (15). Following the general procedure of Weiler, ${ }^{3} \mathrm{NaH}$ (413 mg, 60\% dispersion, $10.43 \mathrm{mmol}$ ) was added slowly to a solution of methyl acetoacetate $(1.00 \mathrm{~g}, 8.62 \mathrm{mmol})$ and THF $(15 \mathrm{~mL})$ at $0{ }^{\circ} \mathrm{C}$. The opaque reaction mixture was stirred for $10 \mathrm{~min}$ at $0{ }^{\circ} \mathrm{C}$, then $n$-BuLi $(3.1 \mathrm{~mL}, 9.5 \mathrm{mmol})$ was added dropwise. The resulting yellow reaction mixture was stirred for an additional $10 \mathrm{~min}$ at $0{ }^{\circ} \mathrm{C}$ before tert-butyl(4-iodobutoxy)dimethylsilane $(2.2$

\footnotetext{
${ }^{3}$ Weiler, L. J. Am. Chem. Soc. 1970, 92, 6702-6704.
} 
$\mathrm{mL}, 8.62 \mathrm{mmol}$ ) was added dropwise. The reaction was allowed to warm to room temperature over $30 \mathrm{~min}$ and then was quenched by the addition of $0.1 \mathrm{~N} \mathrm{HCl}(\sim 2 \mathrm{~mL})$. Silica gel was added, the reaction mixture was concentrated and the residue was purified by chromatography to yield 3-oxo-8-(tertbutyldimethylsiloxy)octanoic acid methyl ester as a colorless liquid (2.41 g, $93 \%)$.

A portion of this liquid (150 mg, $0.49 \mathrm{mmol}$ ) was dissolved in $\mathrm{MeOH}(2 \mathrm{~mL})$ and DOWEX-50 (300 mg) was added. This mixture was stirred at room temperature for $2 \mathrm{~d}$, after which it was filtered and concentrated. The resulting residue was azeotroped with benzene $(3 \mathrm{x}, 25 \mathrm{~mL})$ and the residue was dissolved in $\mathrm{Et}_{2} \mathrm{O}(20 \mathrm{~mL})$. Diketene was added $(60 \mathrm{uL}, 7.4 \mathrm{mmol})$ and the reaction mixture was stirred for $18 \mathrm{~h}$ at room temperature. Silica gel was added, the reaction mixture was concentrated and the residue was purified by chromatography (4:1 hexanes:EtOAc) to afford $81 \mathrm{mg}(60 \%)$ of 15 as a yellow liquid: ${ }^{1} \mathrm{H}$ NMR (400 MHz, $\mathrm{CDCl}_{3}$ ) $\square 4.13$ (app t, $\left.J=6.4 \mathrm{~Hz}, 2 \mathrm{H}\right), 3.73(\mathrm{~s}, 3 \mathrm{H}), 2.55$ (app t, $\left.J=4.0 \mathrm{~Hz}, 2 \mathrm{H}\right), 2.26$ (s, $3 \mathrm{H}), 1.67-1.58(\mathrm{~m}, 8 \mathrm{H}), 1.38-1.34(\mathrm{~m}, 2 \mathrm{H}) ;{ }^{13} \mathrm{C} \mathrm{NMR}\left(100 \mathrm{MHz}, \mathrm{CDCl}_{3}\right) \square 202.4,167.6,167.1,65.1$, 52.3, 50.0, 49.0. 42.7, 30.1, 28.2, 25.2, 22.9; IR (thin film) 2941, 1744. 1713, 1360, 1150, 1017, $999 \mathrm{~cm}^{-1}$; HRMS $\left(\mathrm{CI}^{+}\right) \mathrm{m} / z 273.1333$ (273.1338 calcd for $\mathrm{C}_{13} \mathrm{H}_{21} \mathrm{O}_{6}, \mathrm{MH}^{+}$).

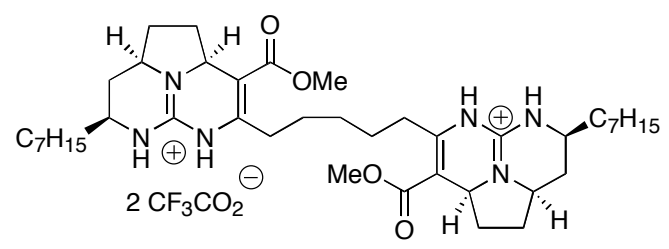

General procedure for double tethered Biginelli cyclizations. Preparation of $\left(2 \mathrm{a} S, 7 S, 8 \mathrm{a} R, 2 \mathrm{a}^{\prime} S, 7 S^{\prime}, 8 \mathrm{a}^{\prime} R\right)$-7-heptyl-4-[5-(7'-heptyl-1',2',2a',5',6',7',8',8a'-octahydro-5',6',8b'triazaacenaphthylene-3'-carboxylic acid methyl ester 4 '-yl)pentyl]-1,2,2a,5,6,7,8,8a-octahydro5,6,8b-triazaacenaphthylene-3-carboxylic acid methyl ester bistrifluoroacetate (16). Bis- $\square$ ketoester 14 (15 mg, $0.06 \mathrm{mmol}$ ) was added to a stirring mixture of guanidine aldehyde 4 (65 mg, 0.21 mmol), morpholinium acetate (80 mg, $0.6 \mathrm{mmol}), \mathrm{Na}_{2} \mathrm{SO}_{4}(80 \mathrm{mg}, 0.5 \mathrm{mmol})$ and $\mathrm{MeOH}(2 \mathrm{~mL}, 0.1 \mathrm{M})$ (In general, morpholine and $\mathrm{AcOH}$ can be added as liquids or as the morpholinium acetate salt). The reaction mixture was heated to $60{ }^{\circ} \mathrm{C}$ and stirred for $72 \mathrm{~h}$. Concentration and purification of the residue by preparative HPLC (Phenomenex C-18, $16 \mathrm{~mL} / \mathrm{min}, 210 \mathrm{~nm}, 10 \% \mathrm{MeCN}$ : $0.1 \%$ TFA in $\mathrm{H}_{2} \mathrm{O}$ to $90 \%$ MeCN: $0.1 \%$ TFA in $\mathrm{H}_{2} \mathrm{O}$ over $20 \mathrm{~min}$ ) gave $18 \mathrm{mg}$ (35\%) of 16 and $12 \mathrm{mg}(23 \%)$ of $C_{1}$-symmetric isomer 22, both as nearly colorless oils. Characterization data for 16: ${ }^{13} \mathrm{C} \mathrm{NMR}\left(125 \mathrm{MHz}, \mathrm{CD}_{3} \mathrm{OD}\right)$ 166.7, 148.1, 147.5, 102.9, 58.3, 57.2, 51.9, 51.5, 34.9, 34.0, 33.9, 32.9, 31.4, 30.4, 30.2, 29.9, 28.8, 27.5, 26.2, 23.7, 14.4; IR (thin film) 2921, 1686, 1457, 1185, $899 \mathrm{~cm}^{-1}$; HRMS (ESI $\left.{ }^{+}\right) \mathrm{m} / z 707.5226(707.5224$

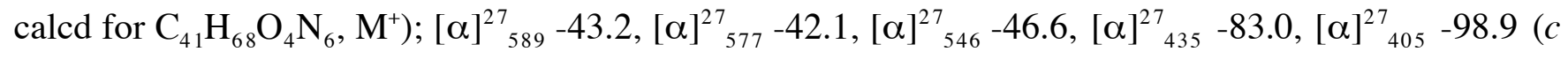
1.0, MeOH). Characterization data for 22: ${ }^{13} \mathrm{C}$ NMR (125 MHz, $\left.\mathrm{CD}_{3} \mathrm{OD}\right)$ 166.7, 148.0, 147.5, 58.2, 57.2, 56.7, 56.1, 53.5, 51.9, 51.8, 51.5, 48.5, 36.0, 34.9, 34.0, 33.9, 33.6, 32.9, 32.8, 32.5, 31.5, 31.4, 30.5, 30.4, 30.3, 29.9, 29.0, 28.8, 27.5, 26.3, 26.2, 23.7, 14.4; HRMS $\left(\mathrm{ESI}^{+}\right) \mathrm{m} / \mathrm{z} 707.5224$ (707.5224 calcd for $\mathrm{C}_{41} \mathrm{H}_{68} \mathrm{O}_{4} \mathrm{~N}_{6}$ ). 


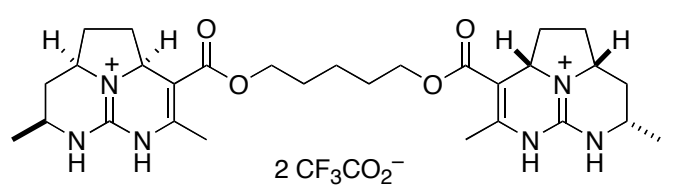

Preparation of $\left(2 \mathrm{a} S, 7 S, 8 \mathrm{a} S, 2 \mathrm{a}^{\prime} S, 7 S^{\prime}, 8 \mathrm{a}^{\prime} S\right)$-7-Methyl-4-methyl-1,2,2a,5,6,7,8,8a-octahydro5,6,8b-triazaacenaphthylene-3-carboxylic acid 4-(7'-methyl-4'-methyl-1',2',2a',5',6',7',8',8a'octahydro-5',6',8b'-triazaacenaphthylene-3'-carboxy)butyl ester bistrifluoroacetate (9). Purification by preparative HPLC (Phenomenex C-18, $210 \mathrm{~nm}, 30 \% \mathrm{MeCN}: 60 \% 0.1 \%$ TFA in $\mathrm{H}_{2} \mathrm{O}$ ) gave $24 \mathrm{mg}(41 \%)$ of the $C_{2}$-symmetric product 9 as a nearly colorless oil: ${ }^{13} \mathrm{C}$ NMR $\left(125 \mathrm{MHz}, \mathrm{CD}_{3} \mathrm{OD}\right)$ $\square$ 166.2, 146.8, 143.9, 102.9, 65.3, 58.2, 57.1, 47.2, 36.1, 34.0, 29.4, 27.6, 23.9, 19.9, 17.8; HRMS (FAB)

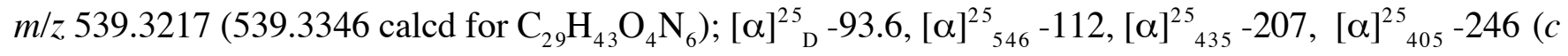
$0.7, \mathrm{MeOH})$.

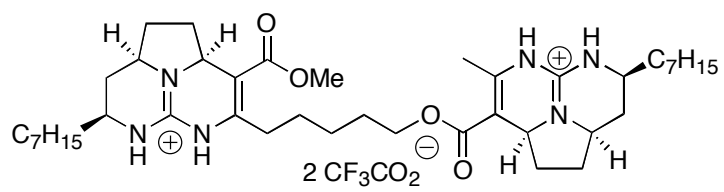

$\left(2 \mathrm{a} S, 7 S, 8 \mathrm{a} R, 2 \mathrm{a}^{\prime} S, 7 S^{\prime}, 8 \mathrm{a}^{\prime} R\right)-4-\left(5-\left(7^{\prime}-\right.\right.$ Heptyl-4-methyl-1',2',2a',5',6',7',8',8a'-octahydro$5^{\prime}, 6$ ',8b'-triaza-acenaphthylene-3'-carboxy)pentyl)-7-heptyl-1,2,2a,5,6,7,8,8a-octahydro-5,6,8btriaza-acenaphthylene-3-carboxylic acid methyl ester bistrifluoroacetate (17). Purification by preparative HPLC (Phenomenex C-18, $16 \mathrm{~mL} / \mathrm{min}, 210 \mathrm{~mm}, 10 \% \mathrm{MeCN}$ : 0.1\% TFA in $\mathrm{H}_{2} \mathrm{O}$ to $90 \%$ MeCN: 0.1\% TFA in $\mathrm{H}_{2} \mathrm{O}$ over $20 \mathrm{~min}$ ) gave $26 \mathrm{mg}$ (39\%) of 17 and $24 \mathrm{mg}$ (37\%) of a stereoisomer that was contaminated with $\mathbf{1 7}$, both as nearly colorless oils. Characterization data for $17:{ }^{13} \mathrm{C} \mathrm{NMR}(125 \mathrm{MHz}$, $\left.\mathrm{CD}_{3} \mathrm{OD}\right) \square 166.7,166.6,148.1,147.4,147.3,144.7,103.5,102.6,65.4$ (2C), 58.3, 58.25, 57.21, 57.2, 51.9, 51.5, 34.9, 33.9, 33.9, 32.9, 31.4, 30.5, 30.3, 29.3, 28.7, 27.5, 27.4, 26.8, 26.3, 26.2, 23.7, 17.8, 14.4;

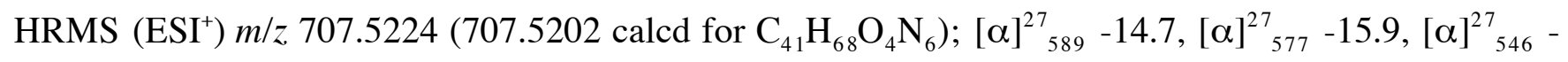
$17.9,[\square]_{435}^{27}-44.1,[\square]_{405}^{27}-57.2$ (c 1.2, MeOH).

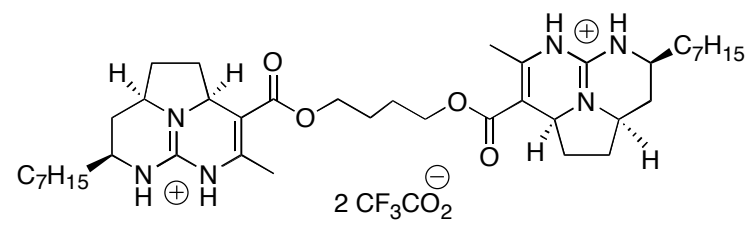

$\left(2 \mathrm{a} S, 7 S, 8 \mathrm{a} R, 2 \mathrm{a}^{\prime} S, 7 S^{\prime}, 8 \mathrm{a}^{\prime} R\right)$-7-Heptyl-4-methyl-1,2,2a,5,6,7,8,8a-octahydro-5,6,8b-triazaacenaphthylene-3-carboxylic acid 4-(7'-heptyl-4'-methyl-1',2',2a',5',6',7',8',8a'-octahydro$5^{\prime}, 6^{\prime}, 8 b^{\prime}$-triazaacenaphthylene-3'-carboxy)-butyl ester bistrifluoroacetate (18) Purification by preparative HPLC (Phenomenex C-18, $16 \mathrm{~mL} / \mathrm{min}, 210 \mathrm{~nm}, 10 \% \mathrm{MeCN}$ : $0.1 \%$ TFA in $\mathrm{H}_{2} \mathrm{O}$ to $90 \%$ 
MeCN: $0.1 \%$ TFA in $\mathrm{H}_{2} \mathrm{O}$ over $20 \mathrm{~min}$ ) gave $20 \mathrm{mg}(42 \%)$ of the $C_{2}$-symmetric product 18 and $10 \mathrm{mg}$ (22\%) of a $C_{1}$-symmetric product that was contaminated with $\mathbf{1 8}$, both as nearly colorless oils. Characterization data for 18: ${ }^{13} \mathrm{C}$ NMR (125 MHz, $\left.\mathrm{CD}_{3} \mathrm{OD}\right) \square 166.5,147.4,144.2$, 103.2, 65.2, 58.4, 57.2, 51.2, 34.9, 33.9, 32.9, 30.4, 30.3, 27.5, 26.6, 26.2, 23.7, 17.8, 14.4; IR (thin film) 2929, 1686, 1200, 1077 ,

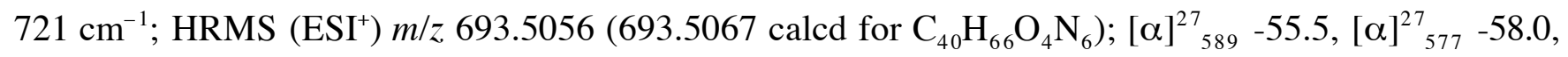
$[\square]_{546}^{27}-61.9,[\square]_{435}^{27}-86.3,[\square]_{405}^{27}-95.8(c 0.5, \mathrm{MeOH})$.

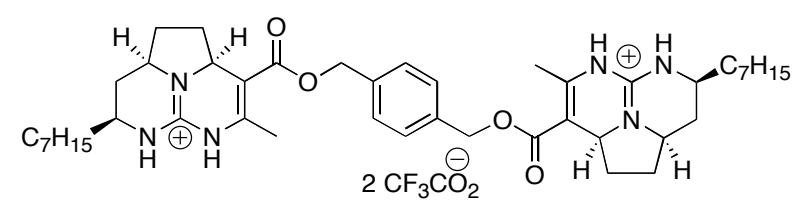

$\left(2 \mathrm{a} S, 7 S, 8 \mathrm{a} R, 2 \mathrm{a}^{\prime} S, 7 S^{\prime}, 8 \mathrm{a}^{\prime} R\right)$-7-Heptyl-4-methyl-1,2,2a,5,6,7,8,8a-octahydro-5,6,8b-triazaacenaphthylene-3-carboxylic acid 4-(7'-heptyl-4'-methyl-1',2',2a',5',6',7',8',8a'-octahydro$5^{\prime}, 6{ }^{\prime}, 8 b^{\prime}$-triazaacenaphthylene-3'-carboxy)methylbenzyl ester bistrifluoroacetate (19). Purification by preparative HPLC (Phenomenex C-18, $16 \mathrm{~mL} / \mathrm{min}, 210 \mathrm{~nm}, 10 \% \mathrm{MeCN}: 0.1 \%$ TFA in $\mathrm{H}_{2} \mathrm{O}$ to $90 \%$ MeCN: $0.1 \%$ TFA in $\mathrm{H}_{2} \mathrm{O}$ over $20 \mathrm{~min}$ ) gave $13 \mathrm{mg}(25 \%)$ of the $C_{2}$-symmetric product 19 and $7 \mathrm{mg}$ (13\%) of a $C_{1}$-symmetric product that was contaminated with some $\mathbf{1 9}$, both as nearly colorless oils. Characterization data for 19: ${ }^{13} \mathrm{C}$ NMR (125 MHz, $\left.\mathrm{CD}_{3} \mathrm{OD}\right) \square$ 163.2, 144.7,141.2, 135.9, 129.8, 103.0, 67.0, 58.3, 57.2, 51.5, 35.0, 33.9, 33.0, 33.4, 30.3, 27.5, 26.2, 23.8, 17.9, 14.5; IR (thin film) 2927, 2858, $1684,1197,1135,718 \mathrm{~cm}^{-1}$; HRMS $\left(\mathrm{ESI}^{+}\right) \mathrm{m} / \mathrm{z} .741 .5063\left(741.5067\right.$ calcd for $\left.\mathrm{C}_{44} \mathrm{H}_{66} \mathrm{O}_{4} \mathrm{~N}_{6}\right)$.

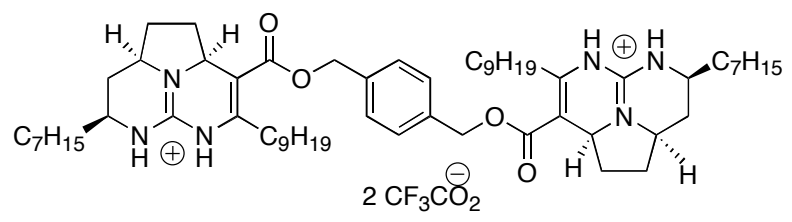

$\left(2 \mathrm{a} S, 7 S, 8 \mathrm{a} R, 2 \mathrm{a}^{\prime} S, 7 S^{\prime}, 8 \mathrm{a}^{\prime} R\right)-7-H e p t y l-4-n o n y l-1,2,2 \mathrm{a}, 5,6,7,8,8 \mathrm{a}-0 c t a h y d r o-5,6,8 \mathrm{~b}-$ triazaacenaphthylene-3-carboxylic acid 4-(7'-heptyl-4'-nonyl-1',2',2a',5',6', 7', ,8',8a'-octahydro-5',6',8b'triazaacenaphthylene-3'-carboxy)methylbenzyl ester bistrifluoroacetate (20). This reaction was carried out by the general procedure using trifluoroethanol as the solvent. Purification by preparative HPLC (Phenomenex C-18, $16 \mathrm{~mL} / \mathrm{min}, 210 \mathrm{~nm}, 10 \% \mathrm{MeCN}: 0.1 \%$ TFA in $\mathrm{H}_{2} \mathrm{O}$ to $90 \% \mathrm{MeCN}: 0.1 \%$ TFA in $\mathrm{H}_{2} \mathrm{O}$ over $20 \mathrm{~min}$ ) gave $17 \mathrm{mg}$ (34\%) of the $C_{2}$-symmetric product 20 and $12 \mathrm{mg}(25 \%)$ of a $C_{1^{-}}$ symmetric product that was contaminated with some 20, both as nearly colorless oils. Characterization data for 20: ${ }^{13} \mathrm{C}$ NMR (125 MHz, $\left.\mathrm{CD}_{3} \mathrm{OD}\right) \square 166.0,148.2,147.3,137.6,129.9,103.0,67.1,58.3,57.2,51.5$, 35.0, 34.0, 33.9, 33.0, 32.9, 32.0, 30.6, 30.4 (4C), 30.3, 29.3, 27.5, 26.3, 23.7, 23.7, 14.5, 14.4; IR (thin film) 2932, 2856, 1583, 1479, 1438, $1250 \mathrm{~cm}^{-1}$; HRMS (ESI) $\mathrm{m} / z 483.3817$ (483.3819 calcd for $\left.\mathrm{C}_{60} \mathrm{H}_{98} \mathrm{O}_{4} \mathrm{~N}_{6}\right) ;[\square]^{27}{ }_{589}-38.8,[\square]_{577}^{27}-37.6,[\square]^{27}{ }_{546}-43.6,[\square]^{27}{ }_{435}-96.1,[\square]^{27}{ }_{405}-120(c 0.5, \mathrm{MeOH})$. 


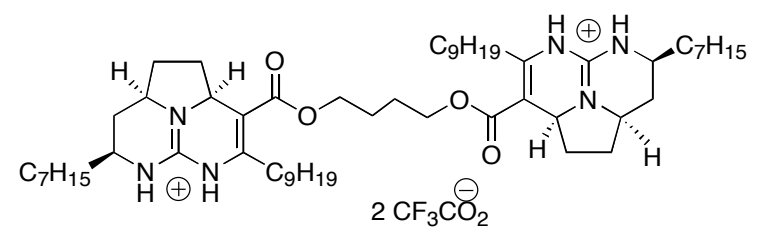

$\left(2 \mathrm{a} S, 7 S, 8 \mathrm{a} R, 2 \mathrm{a}^{\prime} S, 7 S^{\prime}, 8 \mathrm{a}^{\prime} R\right)$-7-Heptyl-4-nonyl-1,2,2a,5,6,7,8,8a-octahydro-5,6,8b-triazaacenaphthylene-3-carboxylic acid 4-(7'-heptyl-4'-nonyl-1',2',2a',5',6',7',8',8a'-octahydro-5',6',8b'triazaacenaphthylene-3'-carboxy)butyl ester bistrifluoroacetate (21). This reaction was carried out by the general procedure using trifluoroethanol as the solvent. Purification by preparative HPLC (Phenomenex C-18, $16 \mathrm{~mL} / \mathrm{min}, 210 \mathrm{~nm}, 10 \% \mathrm{MeCN}$ : 0.1\% TFA in $\mathrm{H}_{2} \mathrm{O}$ to $90 \% \mathrm{MeCN}: 0.1 \%$ TFA in $\mathrm{H}_{2} \mathrm{O}$ over $20 \mathrm{~min}$ ) gave $16 \mathrm{mg}(33 \%)$ of the $C_{2}$-symmetric product 21 and $5 \mathrm{mg}(11 \%)$ of a $C_{1}$-symmetric product that was contaminated with some $\mathbf{2 1}$, both as nearly colorless oils. Characterization data for 21 : ${ }^{13} \mathrm{C}$ NMR (125 MHz, CD 3 OD) $\square 165.8,147.6,146.6,102.3,67.2,57.2,56.3,51.5,43.4,35.0,34.0,33.0,32.9$, $31.8,30.6,30.5,30.4,30.44,30.3,29.2,27.5,26.6,26.3,23.8,23.7,14.4$; IR (thin film) 3352, 2927, 2495, 1671, 1686, 1459, $1202 \mathrm{~cm}^{-1}$; HRMS $\left(\mathrm{ESI}^{+}\right) \mathrm{m} / \mathrm{z} 459.3835$ (459.3819 calcd for $\mathrm{C}_{56} \mathrm{H}_{98} \mathrm{O}_{4} \mathrm{~N}_{6}[\mathrm{M}]^{2+}$ ); $[\square]_{589}^{27}-41.3,[\square]_{577}^{27}-50.7,[\square]_{546}^{27}-72.4,[\square]_{435}^{27}-123.1,[\square]_{405}^{27}-159.2(c 0.5, \mathrm{MeOH})$.

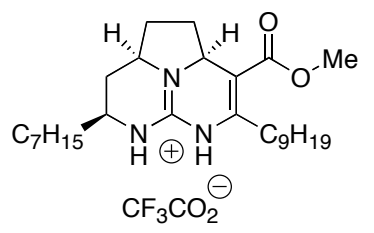

(2aS,7S,8aR)-7-Heptyl-4-nonyl-1,2,2a,5,6,7,8,8a-octahydro-5,6,8b-triazaacenaphthylene-3carboxylic acid methyl ester trifluoroacetate $\left(23 \mathrm{R}=n-\mathrm{C}_{7} \mathrm{H}_{15}\right)$. Purification by preparative HPLC (Phenomenex C-18, $16 \mathrm{~mL} / \mathrm{min}, 210 \mathrm{~nm}, 10 \% \mathrm{MeCN}$ : 0.1\% TFA in $\mathrm{H}_{2} \mathrm{O}$ to $90 \% \mathrm{MeCN}: 0.1 \%$ TFA in $\mathrm{H}_{2} \mathrm{O}$ over $\left.20 \mathrm{~min}\right)$ gave $45 \mathrm{mg}(77 \%)$ of 23 as a nearly colorless oil: ${ }^{1} \mathrm{H}$ NMR (500 $\left.\mathrm{MHz}, \mathrm{CD}_{3} \mathrm{OD}\right)$ $\square 4.59-4.55$ (m, 1H), 3.86-3.82 (m, 1H), 3.77 (s, 3H), 3.58-3.49 (m, 1H), 2.84-2.76 (m, 1H), 2.75-2.66 (m, 1H), 2.61-2.53 (m, 1H), 2.49-2.44 (m, 1H), 2.22-2.17 (m, 1H), 1.74-1.55 (m, 6H), 1.53-1.38 (m, 25H), 0.96-0.92 (m, 6H); ${ }^{13} \mathrm{C}$ NMR (125 MHz, CD $\left.\mathrm{OD}\right) \square 166.7,148.2,147.5,102.8,58.3$, 57.2, 51.9, 51.5, 34.9, 34.0, 33.9, 33.0, 32.9, 31.6, 30.6, 30.4, 30.4, 30.3 (2C), 30.3, 29.2, 27.5, 26.2, 23.7, 23.7, 14.4, 14.3; IR (thin film) 2927, 2858, 1686, 1436, 1202, 1140, 906, $727 \mathrm{~cm}^{-1}$; HRMS (ESI) m/z 446.3764

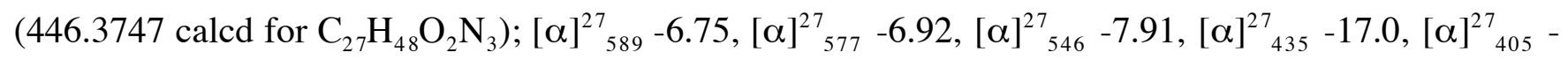
20.1 ( c 3.8, $\mathrm{MeOH})$.

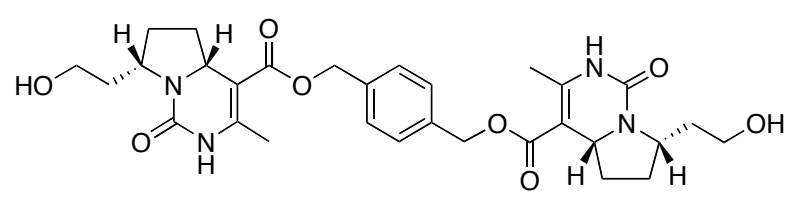


(2S,4aR,2'S,4a'R)-7-(2-Hydroxy-ethyl)-3-methyl-1-oxo-1,2,4a,5,6,7-hexahydropyrrolo[1,2-c]pyrimidine-4-carboxylic acid 4-(7'-(2'-hydroxyethyl)-3'-methyl-1'-oxo$1^{\prime}, 2^{\prime}, 4 a^{\prime}, 5^{\prime}, 6^{\prime}, 7^{\prime}$-hexahydropyrrolo[1',2'-c]pyrimidine-4'-carboxy)-methylbenzyl ester (25). Purification by preparative HPLC (Phenomenex C-18, $16 \mathrm{~mL} / \mathrm{min}, 210 \mathrm{~nm}, 10 \% \mathrm{MeCN}: 0.1 \%$ TFA in $\mathrm{H}_{2} \mathrm{O}$ to $90 \% \mathrm{MeCN}$ : $0.1 \%$ TFA in $\mathrm{H}_{2} \mathrm{O}$ over $20 \mathrm{~min}$ ) gave $21 \mathrm{mg}(69 \%)$ of the $C_{2}$-symmetric product 25 and $7 \mathrm{mg}(23 \%)$ of a less pure sample of a $C_{1}$-symmetric isomer, both as pale yellow films. Characterization data for 25: ${ }^{1} \mathrm{H}$ NMR $\left(500 \mathrm{MHz} \mathrm{CDCl}_{3}\right.$ ) (trace amounts of $\mathrm{MeOH}$ added to solubilize 25) $\square 7.61$ (br s, 1H), 7.41 (s, 2H), $5.25(\mathrm{~d}, J=2.5 \mathrm{~Hz}, 1 \mathrm{H}), 5.18(\mathrm{~d}, J=2.5 \mathrm{~Hz}, 1 \mathrm{H}), 4.33-4.30(\mathrm{~m}, 1 \mathrm{H})$, 4.21-4.17 (m, 1H), 3.65-3.55 (m, 1H), $2.25(\mathrm{~s}, 3 \mathrm{H}), 2.13-2.08(\mathrm{~m}, 1 \mathrm{H}), 1.93-1.65(\mathrm{~m}, 3 \mathrm{H})$; ${ }^{13} \mathrm{C}$ NMR $\left(125 \mathrm{MHz}, \mathrm{CDCl}_{3}\right) \square 165.5,154.4,148.8,136.2,128.7,102.5,65.7,59.2,58.5,52.4,50.9,39.5,30.8,29.9$, 18.4; IR (thin film) 2950, 1676, 1630, 1437, 1313, 1251, 1112, $1074 \mathrm{~cm}^{-1}$; HRMS (ESI) $\mathrm{m} / z, 605.2572$

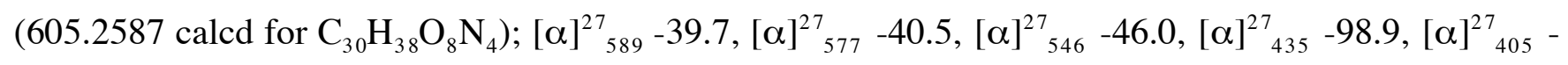
110.1 ( c 1, MeOH).

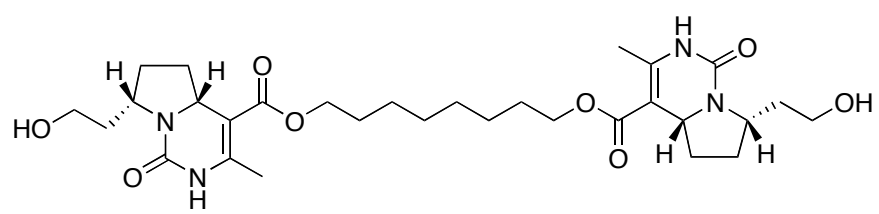

(2S,4aR,2'S,4a'R)-7-(2-Hydroxyethyl)-3-methyl-1-oxo-1,2,4a,5,6,7-hexahydro-pyrrolo[1,2c]pyrimidine-4-carboxylic acid 4-(7'-(2'-hydroxyethyl)-3'-methyl-1'-oxo-1',2',4a',5',6',7'hexahydropyrrolo[1',2'-c]pyrimidine-4'-carboxy)octyl ester (27). Purification by preparative HPLC (Phenomenex C-18, $16 \mathrm{~mL} / \mathrm{min}, 210 \mathrm{~nm}, 10 \% \mathrm{MeCN}: 0.1 \%$ TFA in $\mathrm{H}_{2} \mathrm{O}$ to $90 \% \mathrm{MeCN}: 0.1 \%$ TFA in $\mathrm{H}_{2} \mathrm{O}$ over $\left.20 \mathrm{~min}\right)$ gave $20 \mathrm{mg}(61 \%)$ of the $C_{2}$-symmetric product 27 and $7 \mathrm{mg}(20 \%)$ of a less pure sample of a $C_{1}$-symmetric isomer, both as pale yellow films the former of which slowly solidified upon standing at $0{ }^{\circ} \mathrm{C}$. Characterization data for $27:{ }^{1} \mathrm{H}$ NMR $\left(500 \mathrm{MHz}, \mathrm{CDCl}_{3}\right) \square 7.54(\mathrm{~m}, 1 \mathrm{H}), 4.31(\mathrm{ddd}, J=$ 11.2, 5.0, 1.2 Hz, 1H), 4.23-4.16 (m, 2H), 4.16-4.11 (m, 1H), 3.70-3.58 (m, 2H), 2.58-2.53 (m, 1H), 2.28 (s, 3H), 2.19-2.10 (m, 1H), 1.95-1.87 (m, 1H), 1.82-1.76 (m, 3H), 1.76-1.68 (m, 4H), 1.39-1.37 (m, 4H); ${ }^{13} \mathrm{C} \mathrm{NMR}\left(125 \mathrm{MHz} \mathrm{CDCl}_{3}\right) \square 165.9,154.5,148.1,103.0,64.2,59.2,58.2,52.4,39.6,30.9,29.9,29.1$, 28.7, 26.0, 18.2; IR (thin film) 3236, 2927, 2858, 1738, 1645, 1452, 1321, 1251, $1081 \mathrm{~cm}^{-1}$; HRMS (ESI $)$

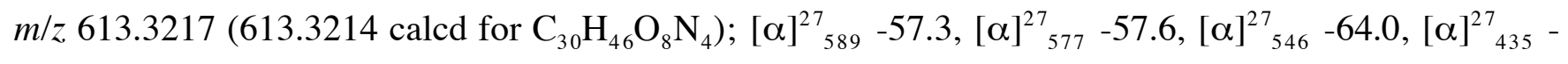
$145.4,[\square]_{405}^{27}-188.7(c 0.5, \mathrm{MeOH})$. 
Supporting Information Cohen, Collins and Overman, September 16, 2003

${ }^{1} \mathrm{H}$ and ${ }^{13} \mathrm{C}$ NMR Spectra

\author{
Q. [-Ketoesters
}




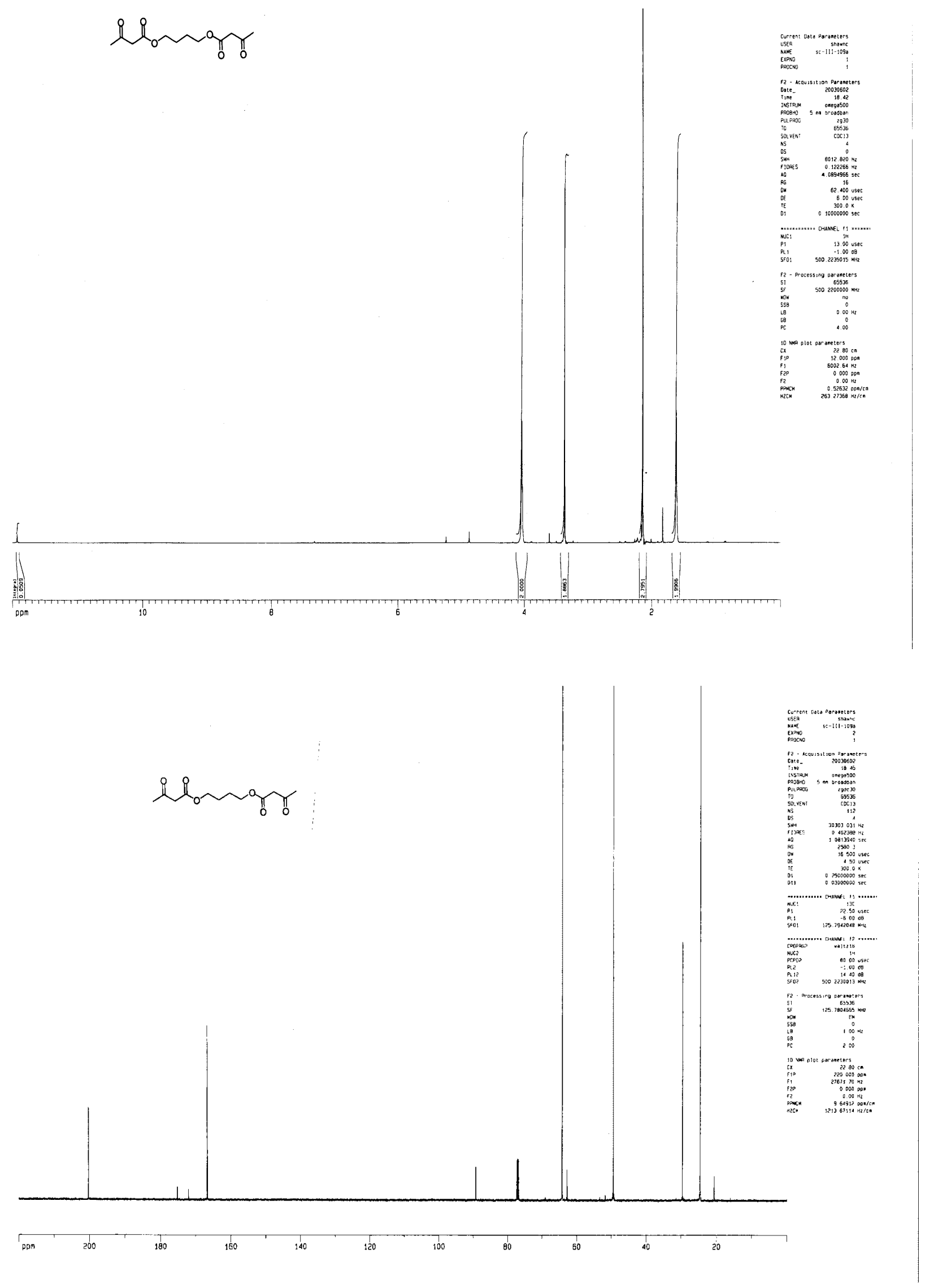




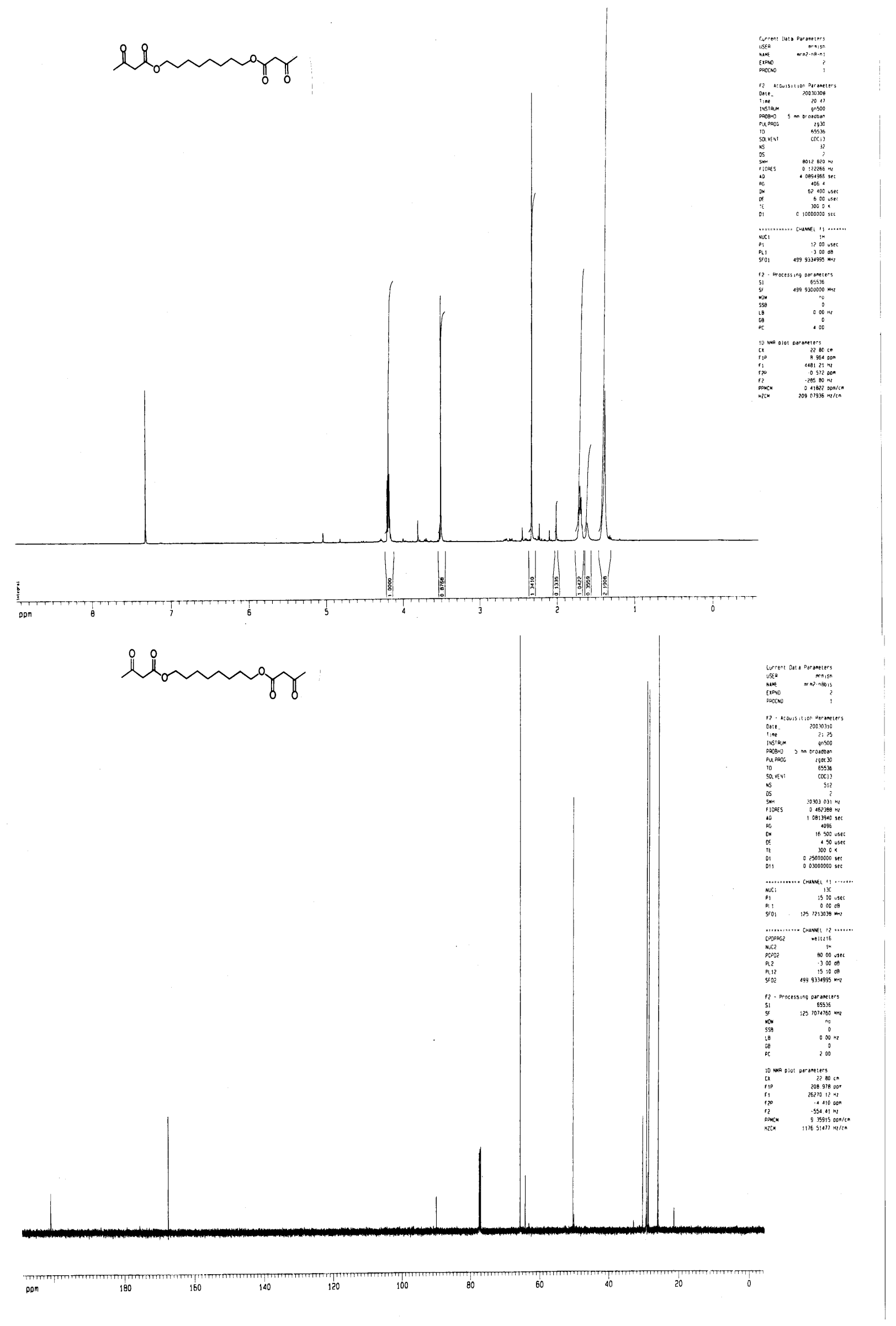



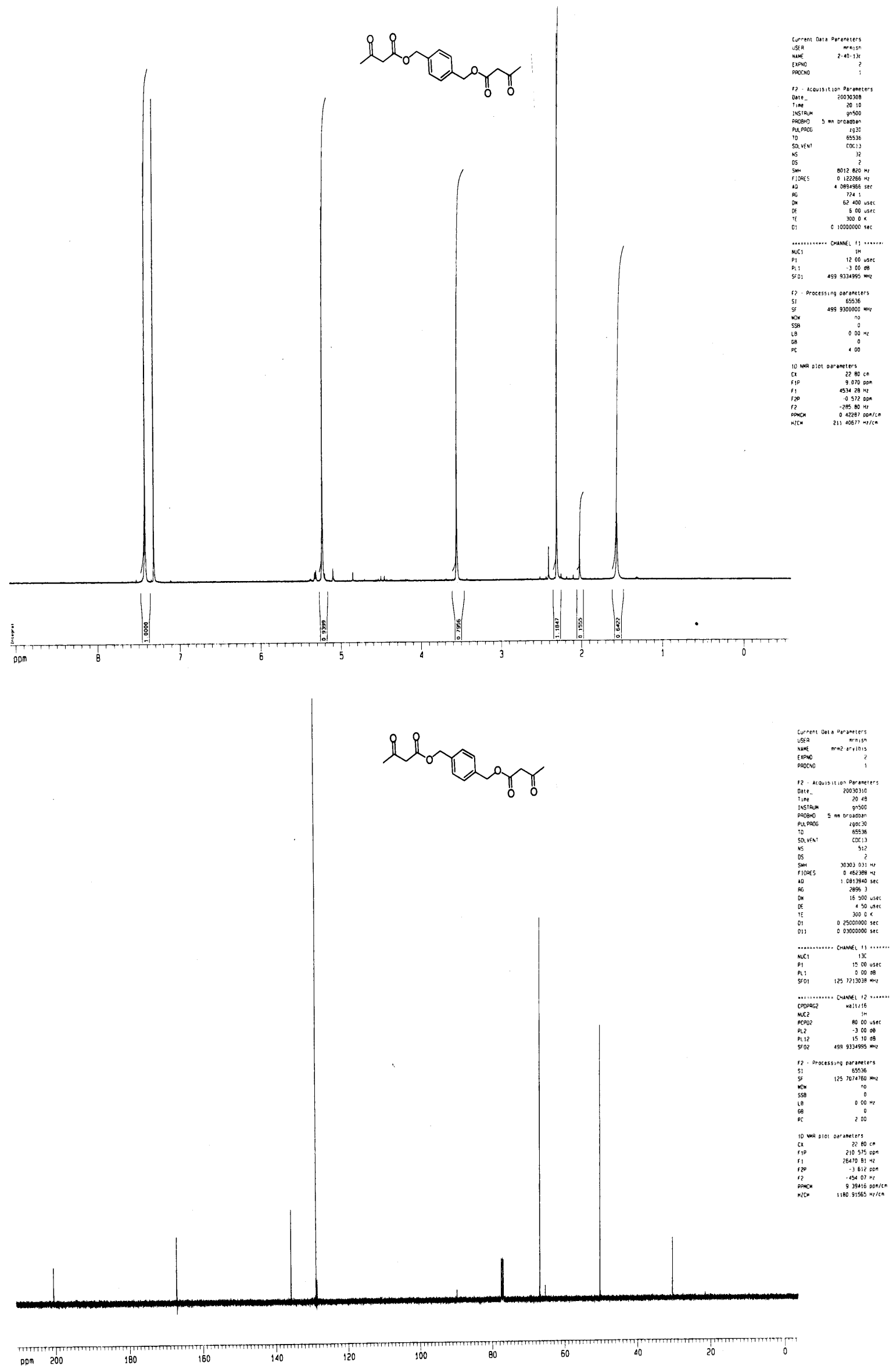


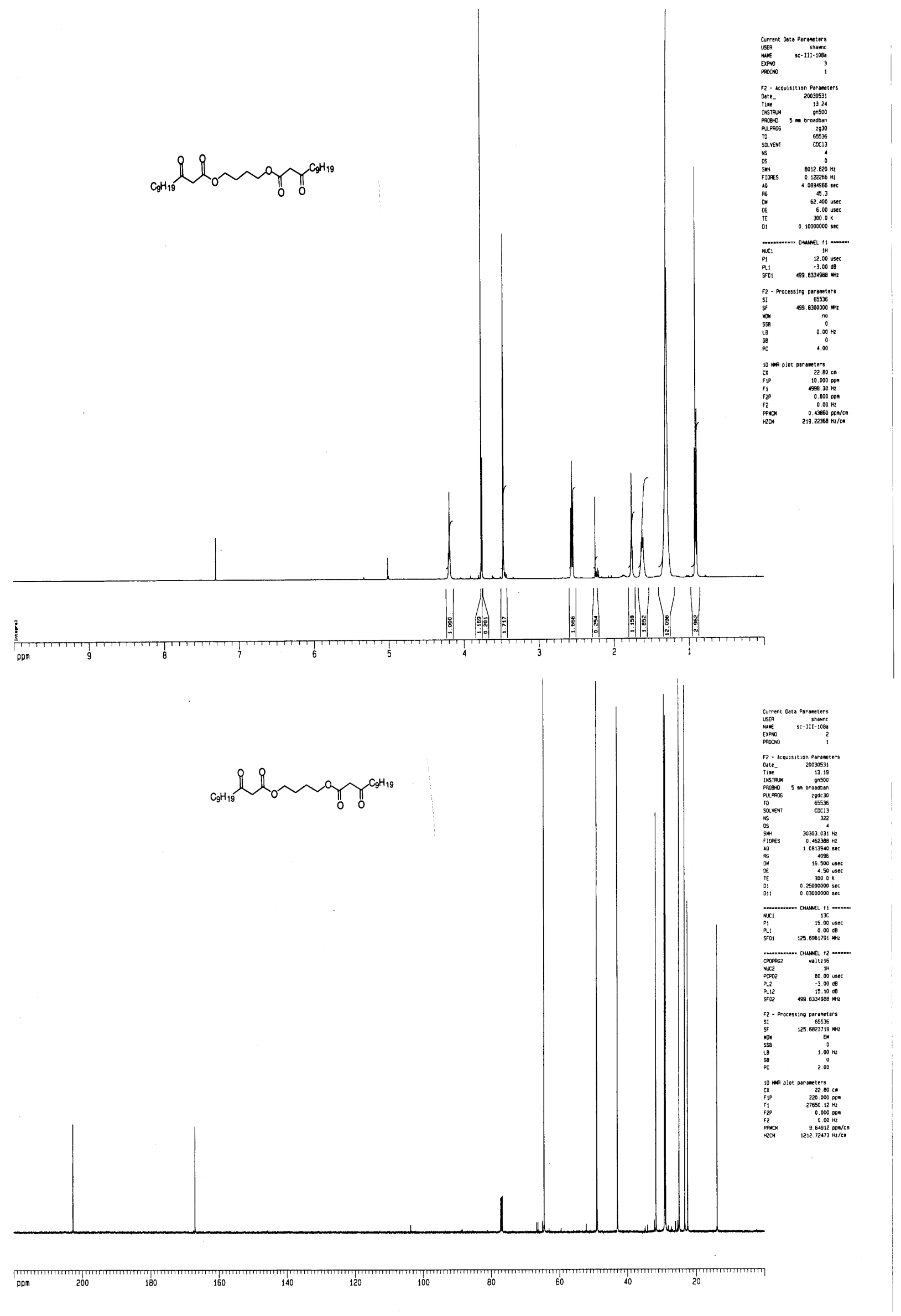




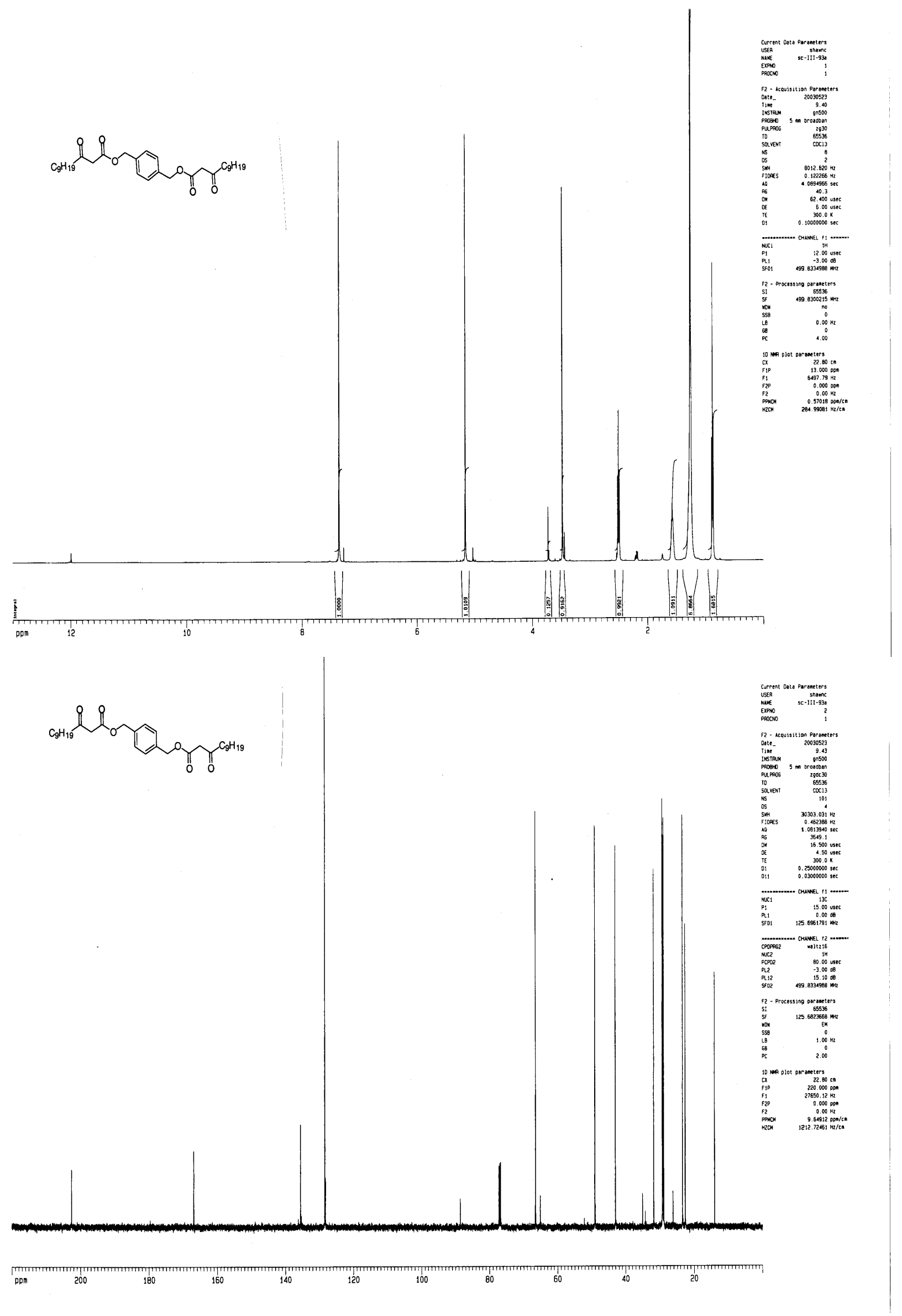




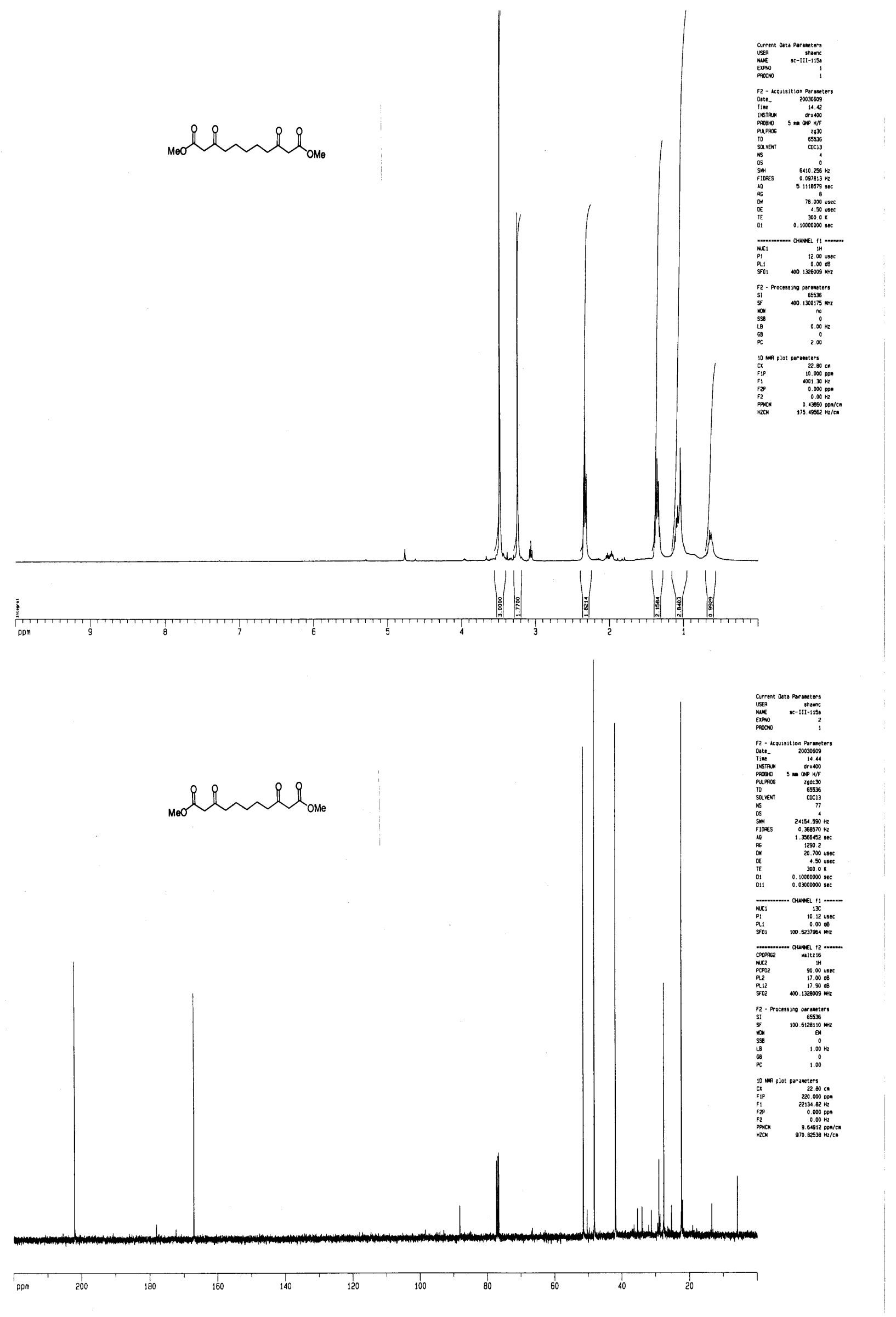



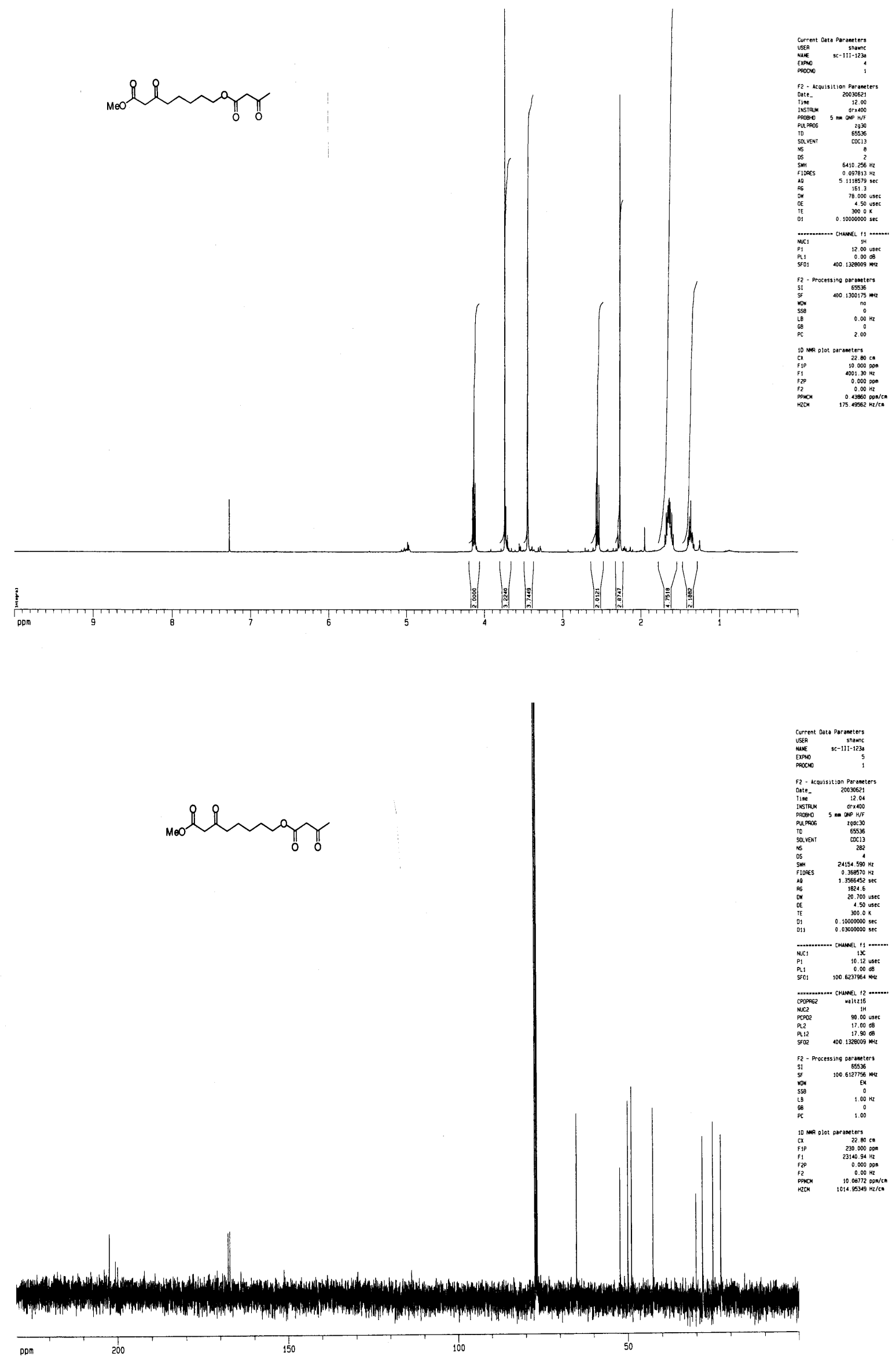


\section{${ }^{1} \mathrm{H}$ and ${ }^{13} \mathrm{C}$ NMR Spectra}

\section{๑. $C_{2}$-Symmetric Bisguanidine and Bisureas}




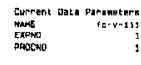

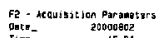

(1)

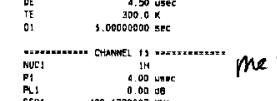

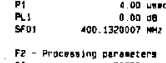

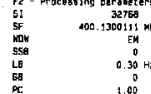

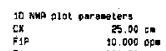

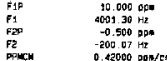

Pret

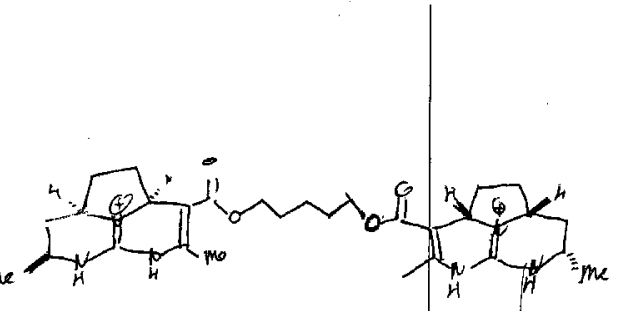

要

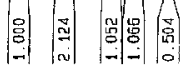

Mu Mullit

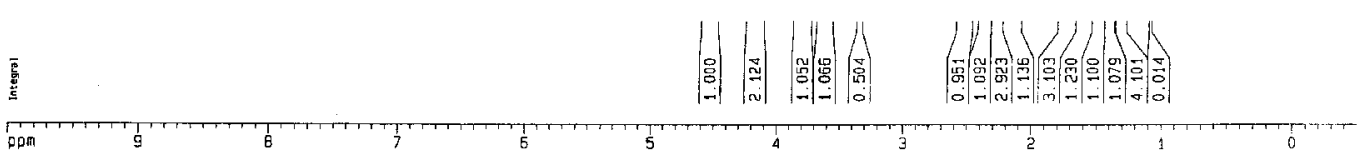

s
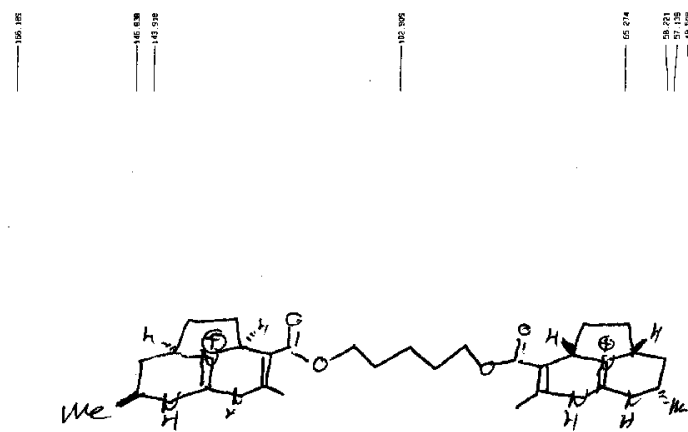

$-2 \mathrm{CF}^{\circ} \mathrm{C}_{2}^{-}$
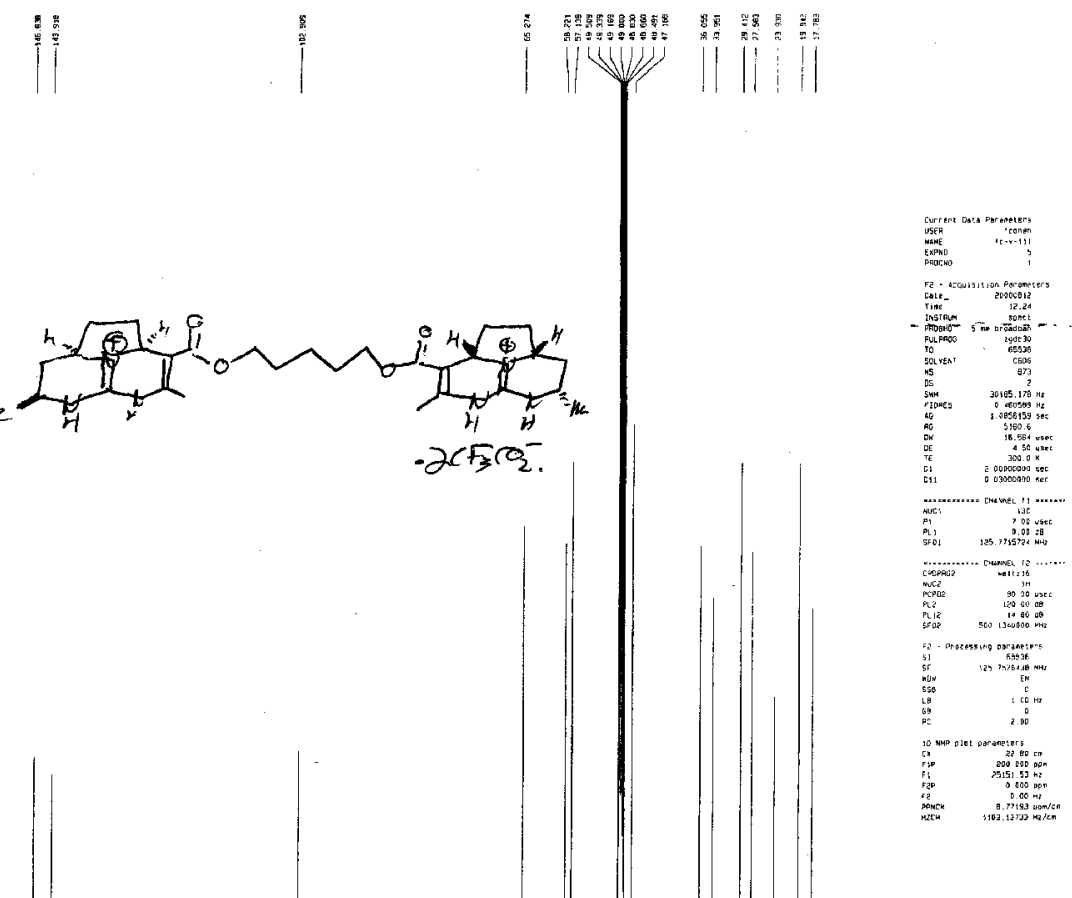


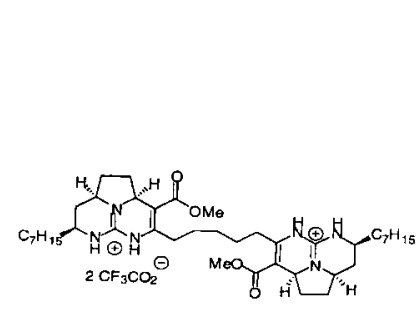

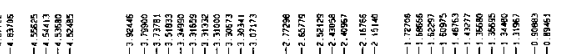

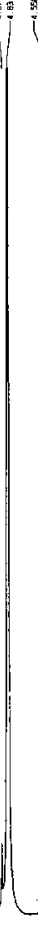

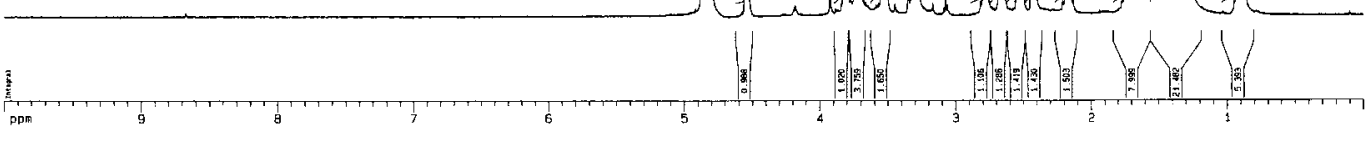

$13 \mathrm{C}$ spectrum with $1 \mathrm{H}$ decoupling
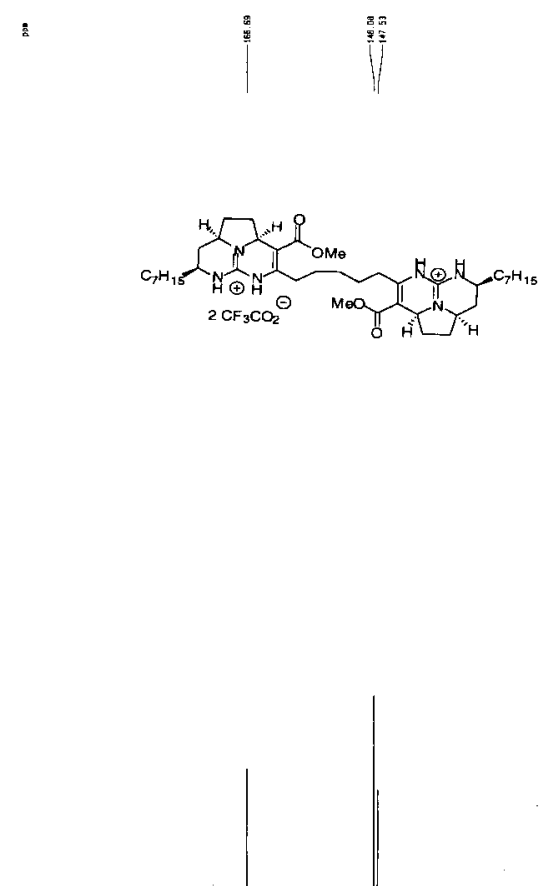


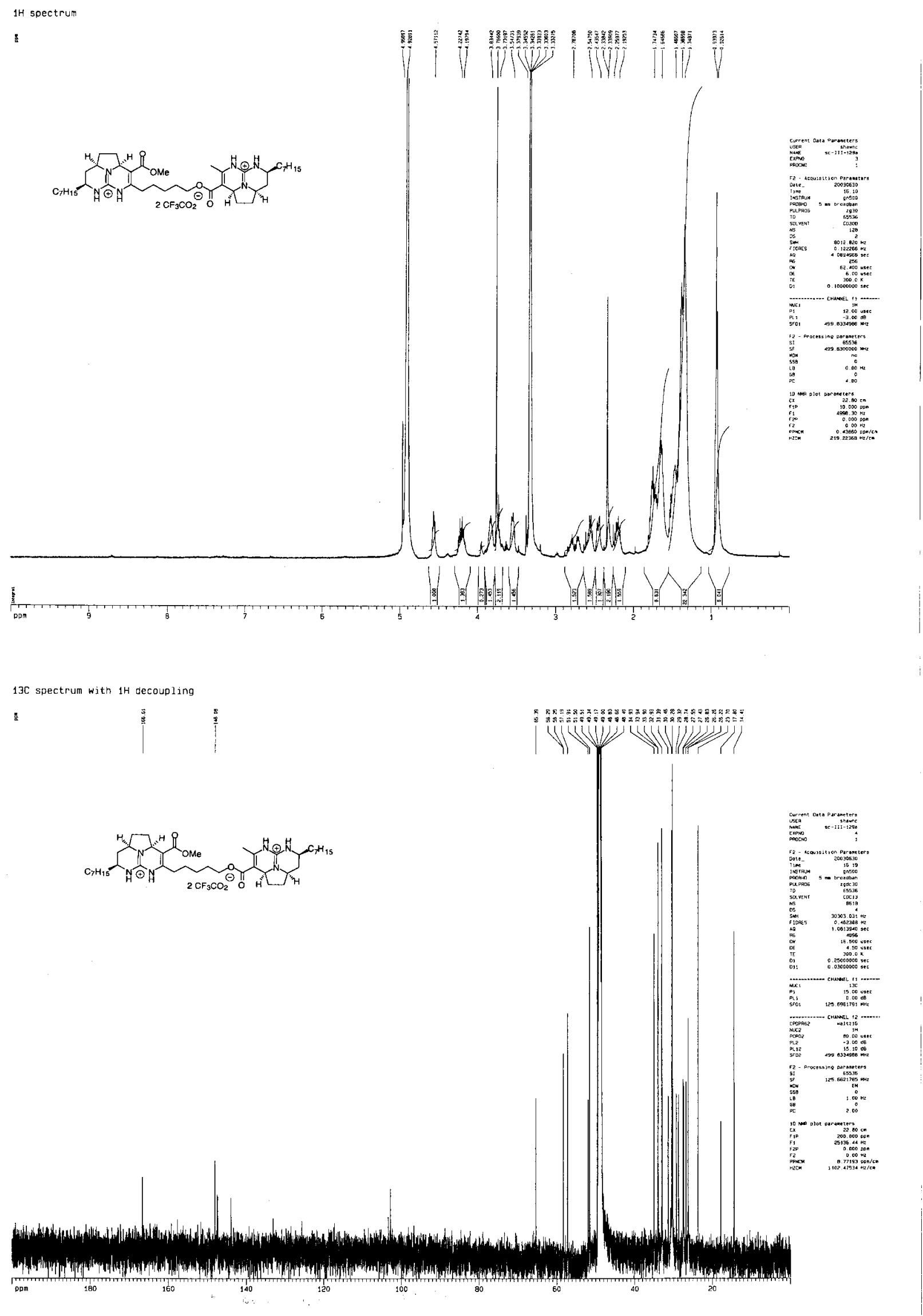




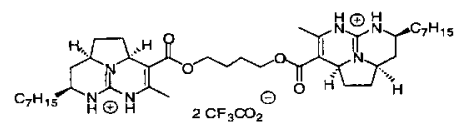

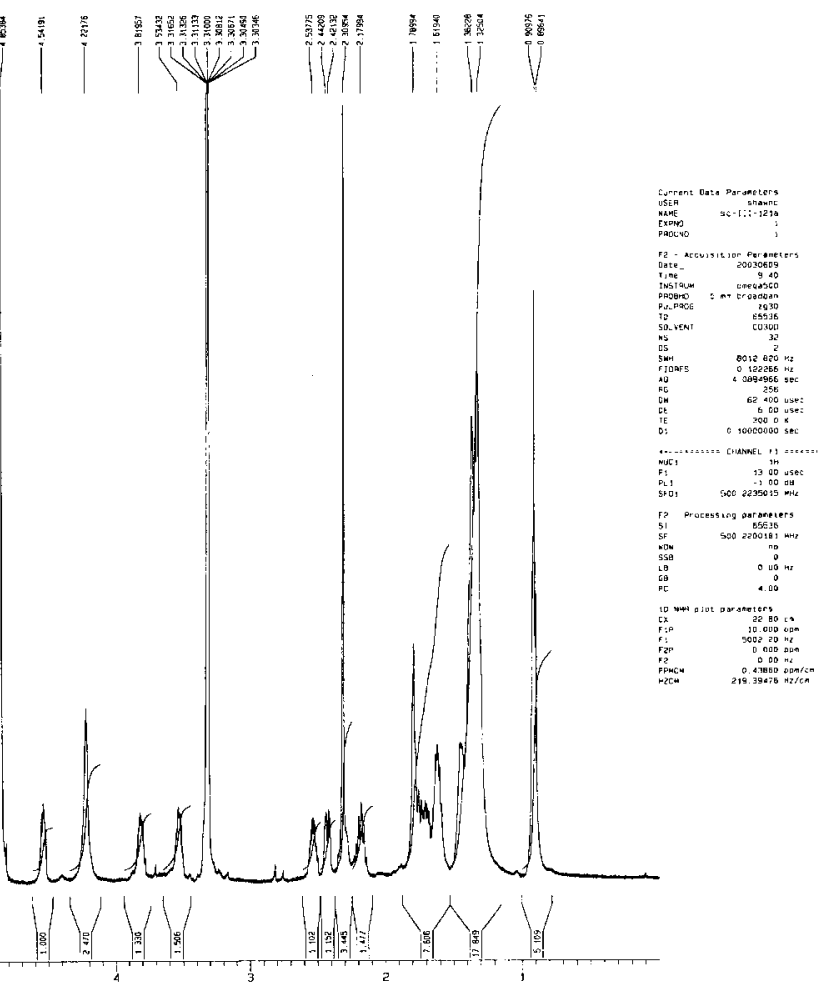

13 C spectrum with $1 \mathrm{H}$ decoupling

5

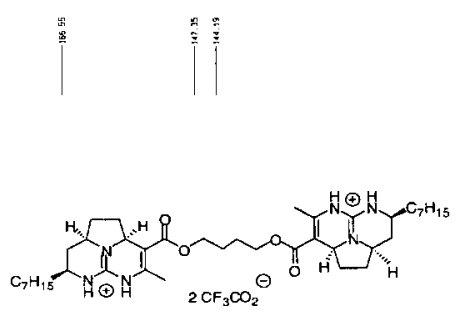

\section{$\overbrace{}^{20}$}

|

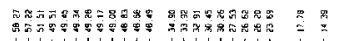

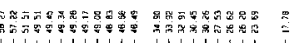

$$
20 \mathrm{H} \quad 2 \mathrm{CF}_{3} \mathrm{CO}_{2}
$$
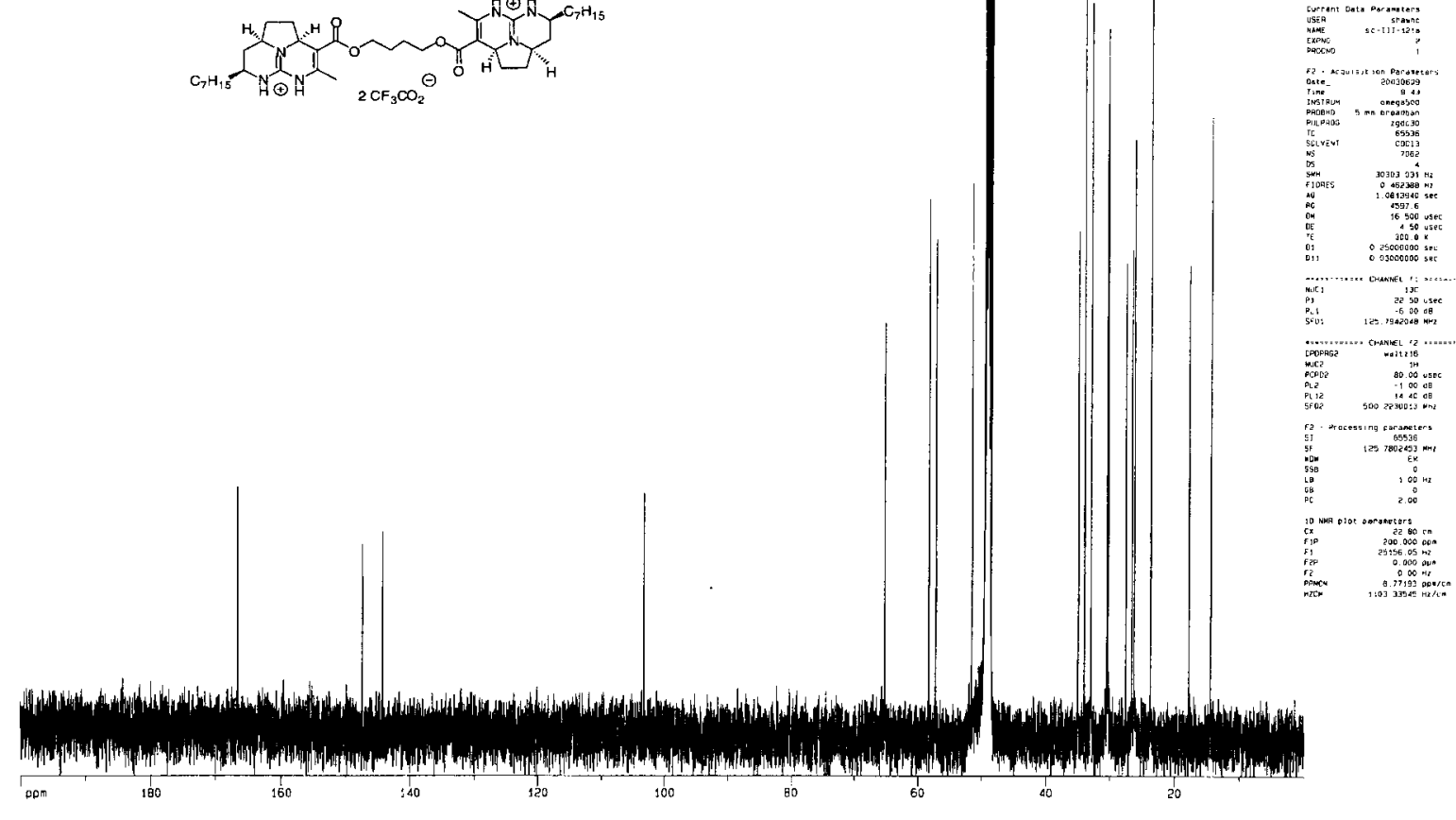
1 spectrum

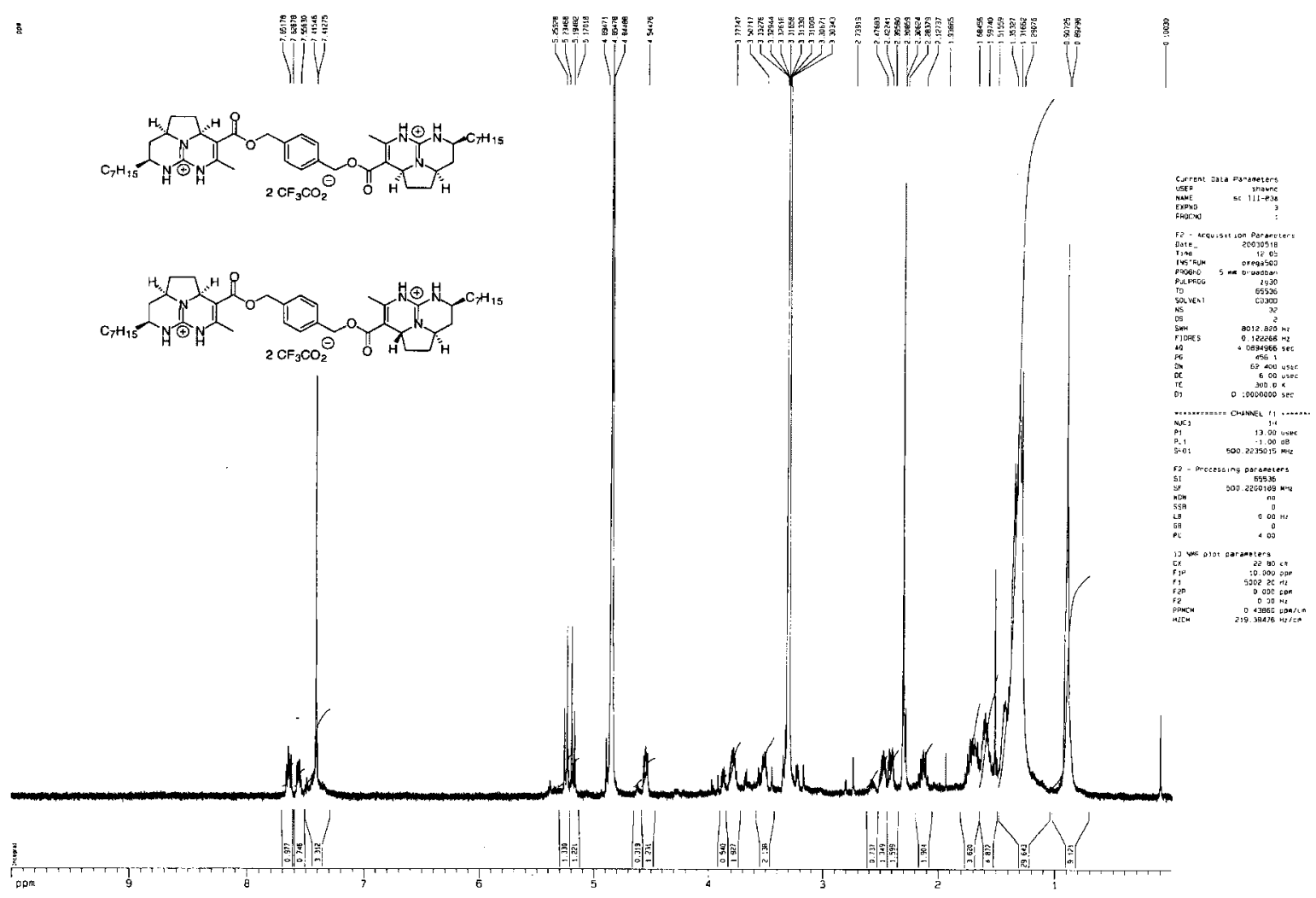

13C soectrum with $1 H$ decoupling

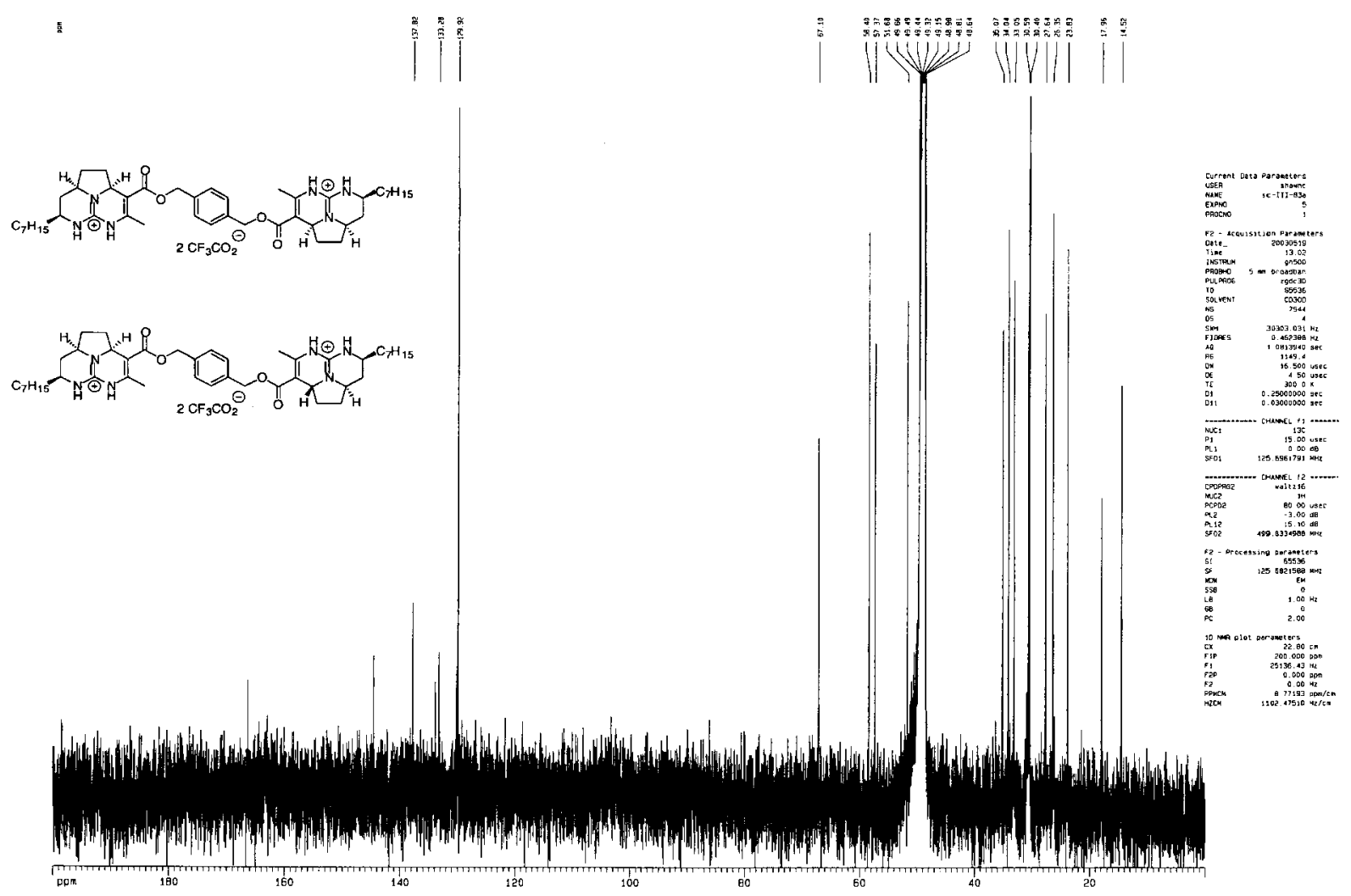




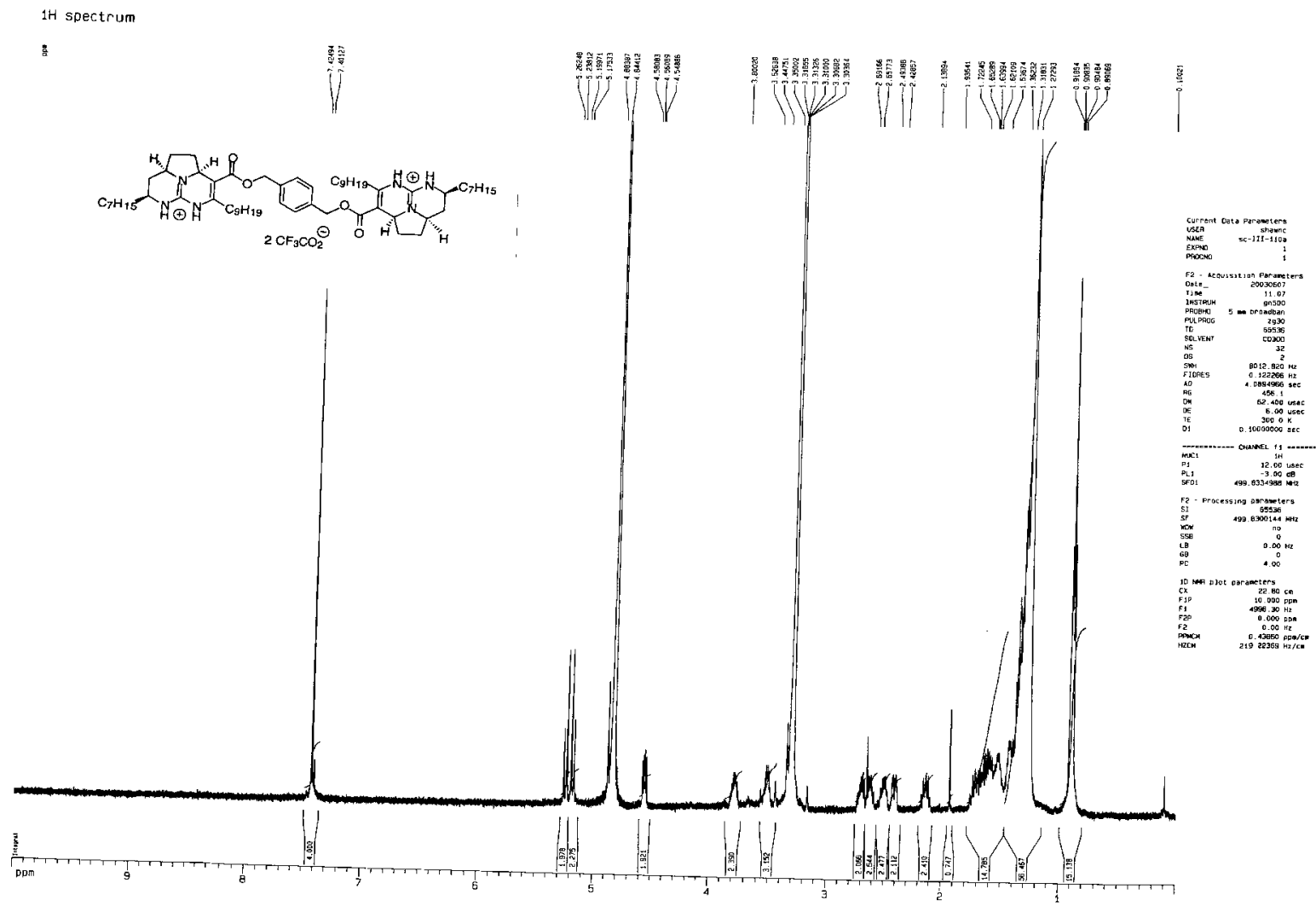

IC spectrum with $1 \mathrm{H}$ decoupling
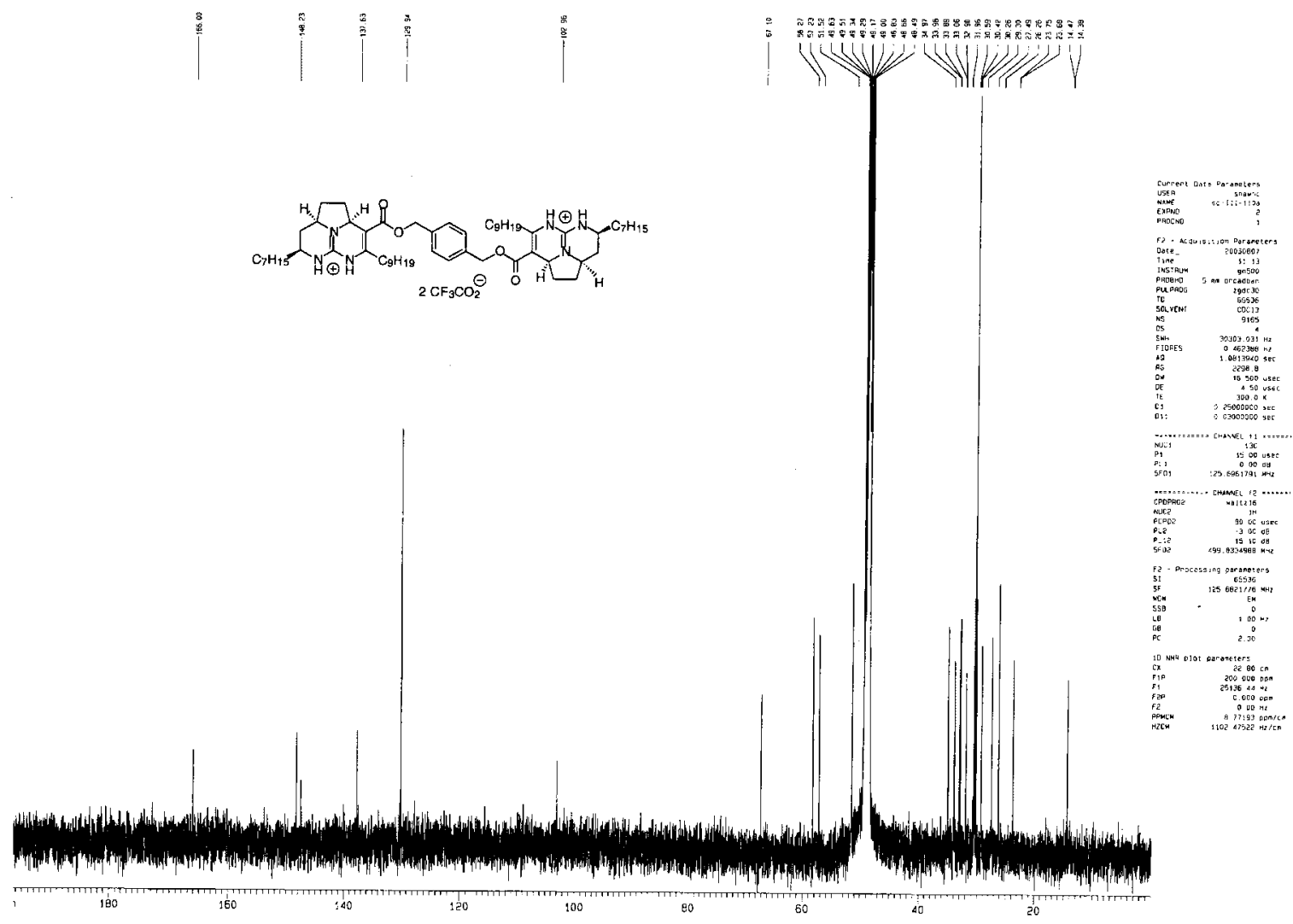


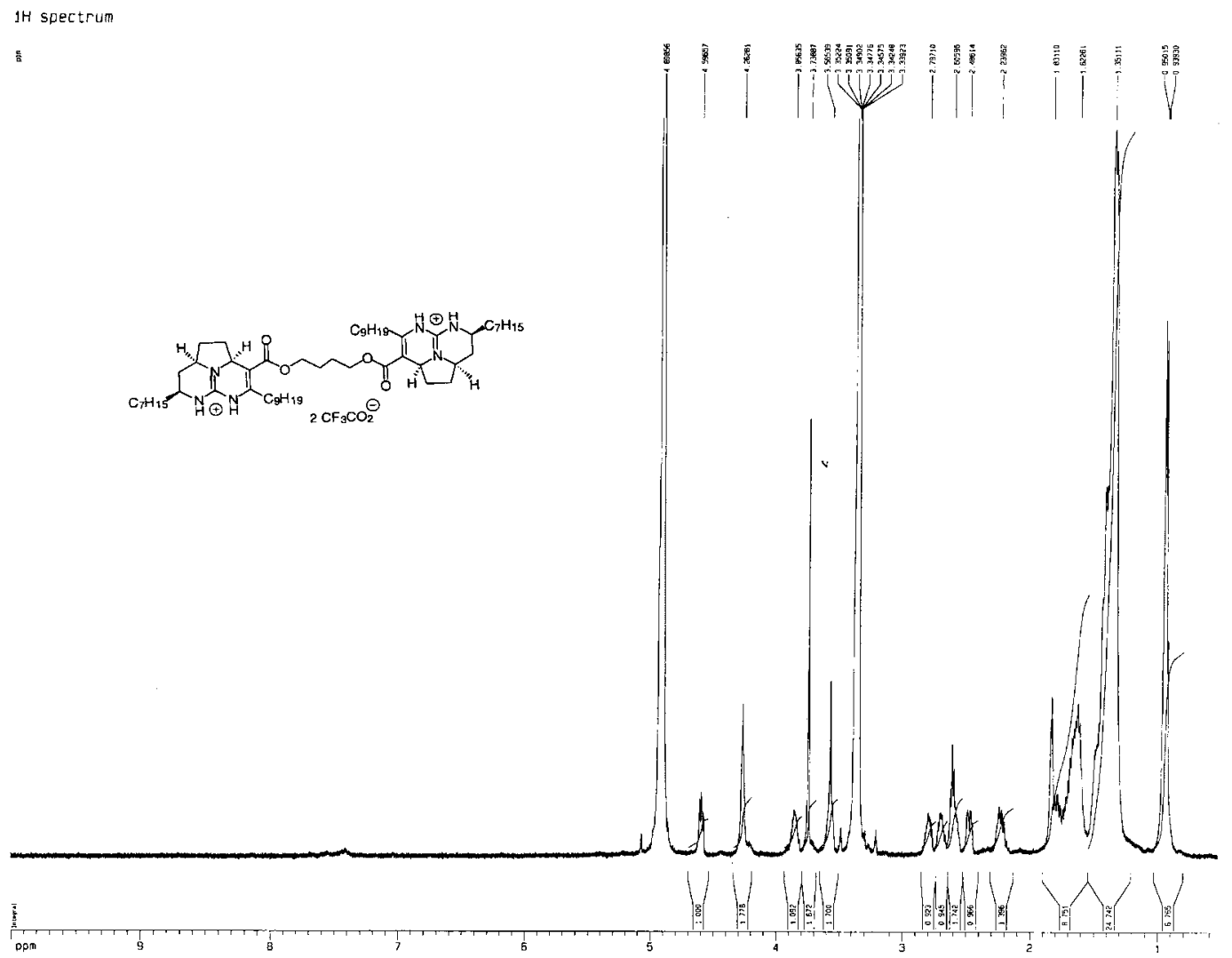

$13 C$ spectrum with $2 H$ decoupling
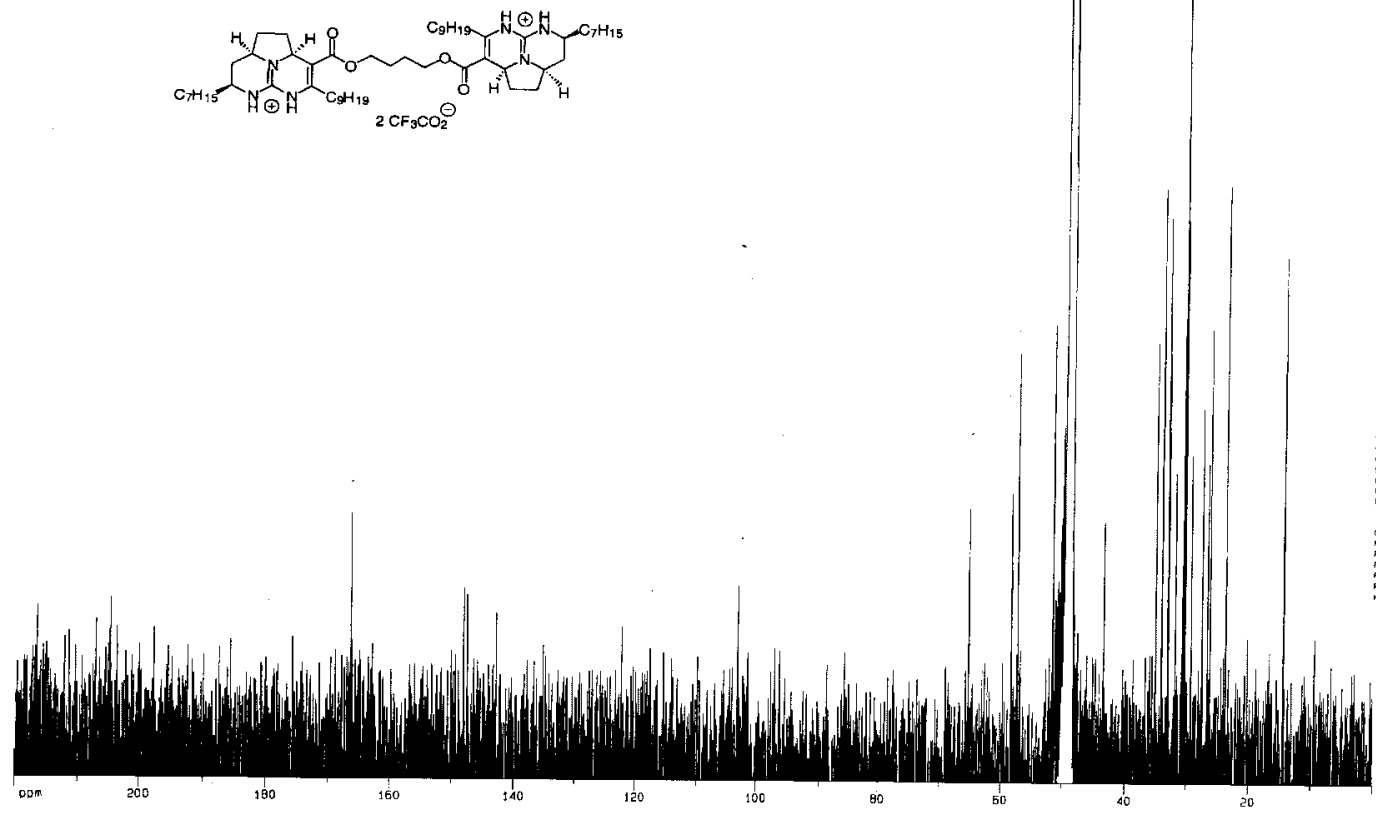


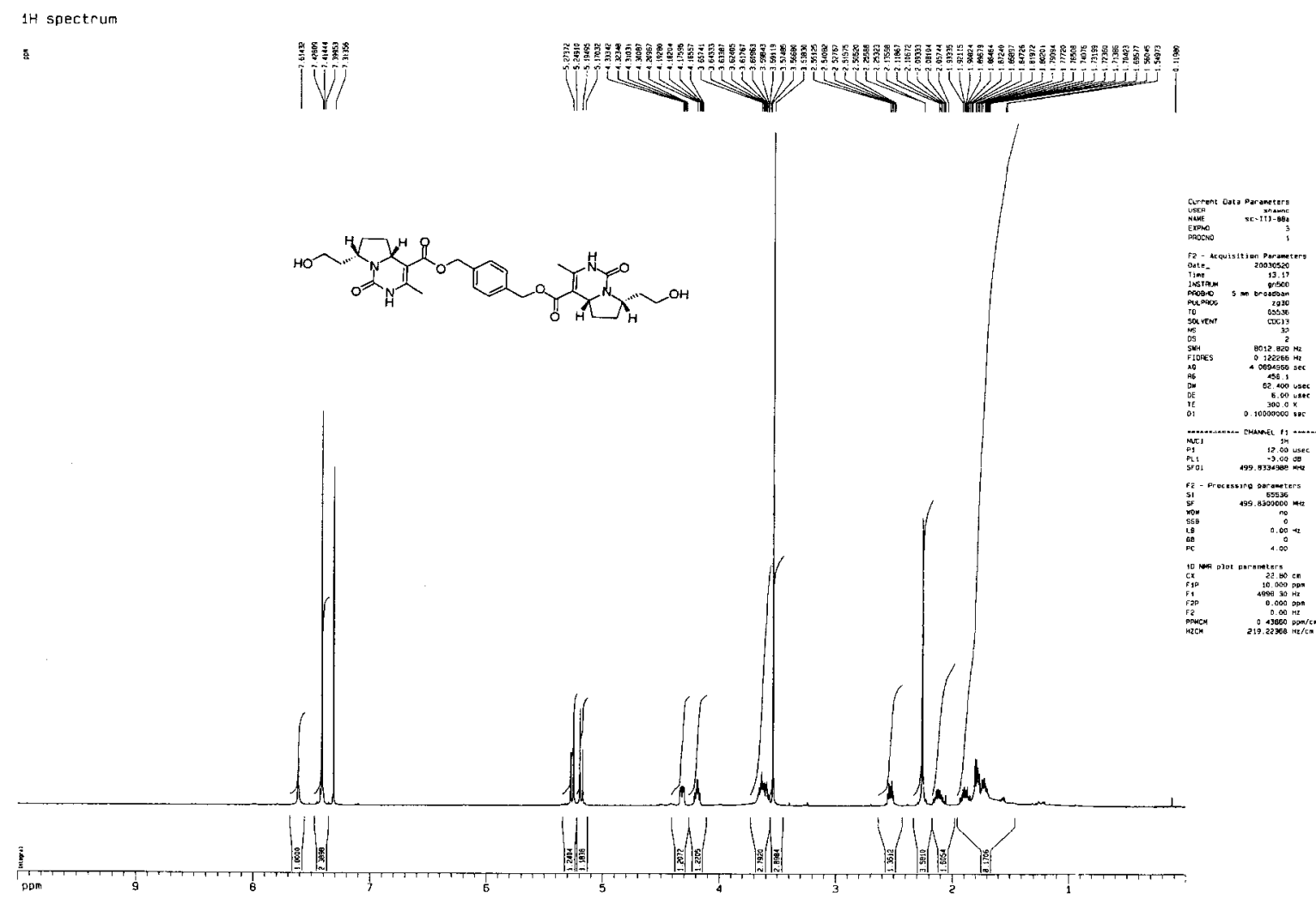

$13 \mathrm{C}$ spectrum with $1 \mathrm{H}$ decoupling

(2)

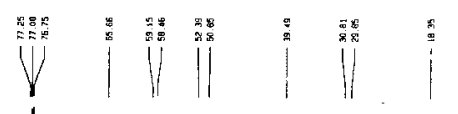

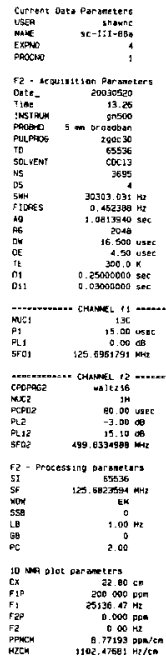

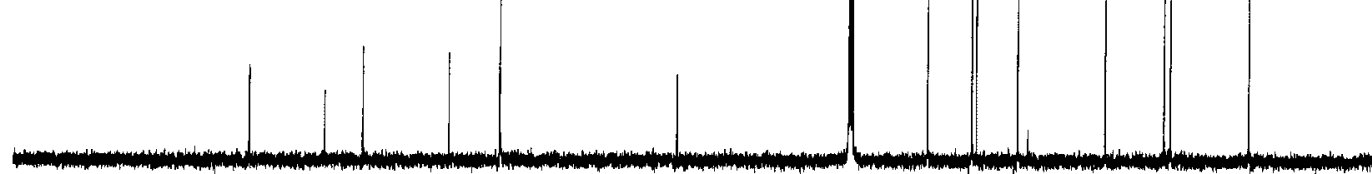

Tpen 160

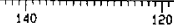

${ }_{120}$ ${ }_{80}$

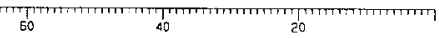




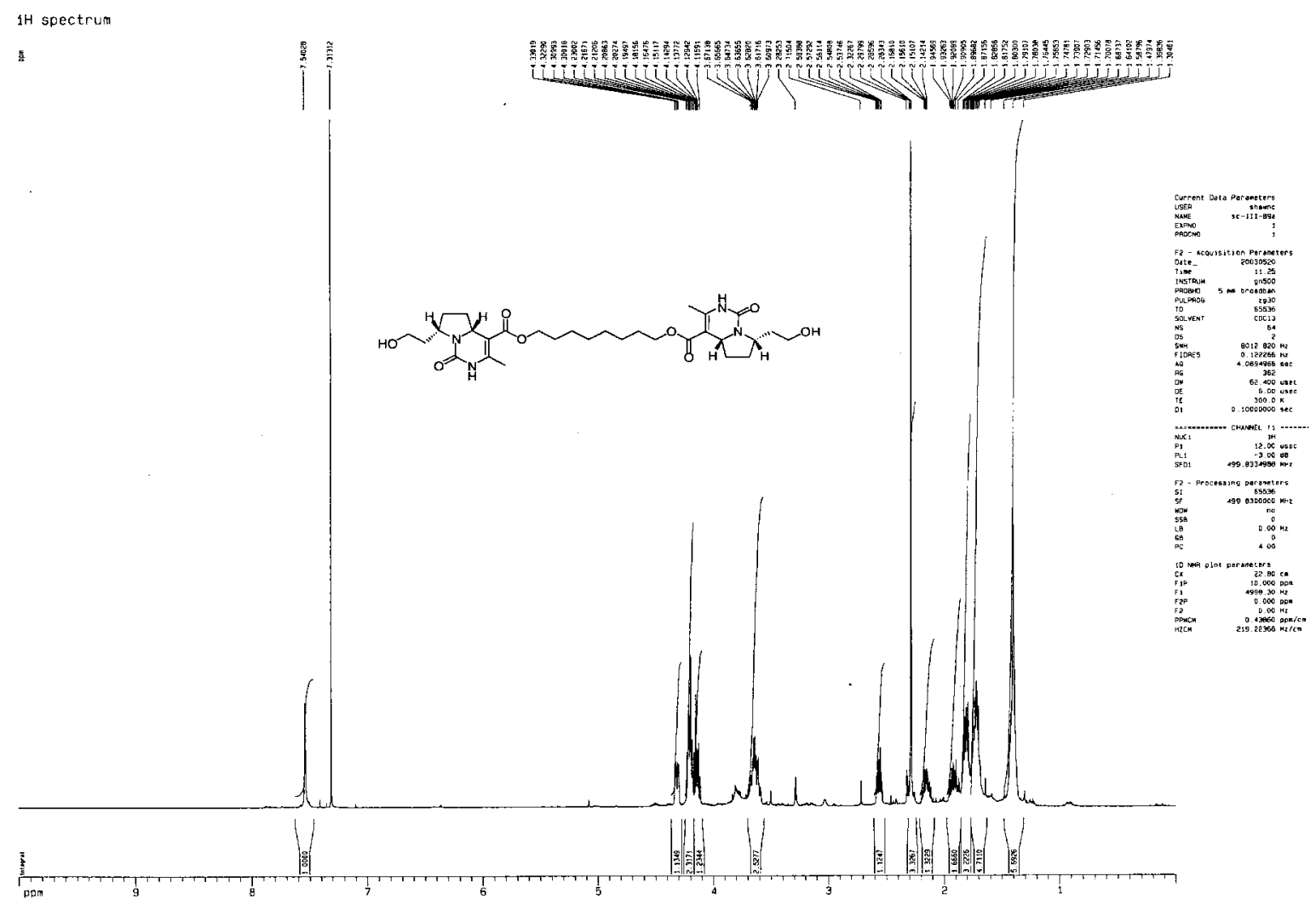

$13 \mathrm{C}$ spectrum with $1 \mathrm{H}$ decoupling

๕

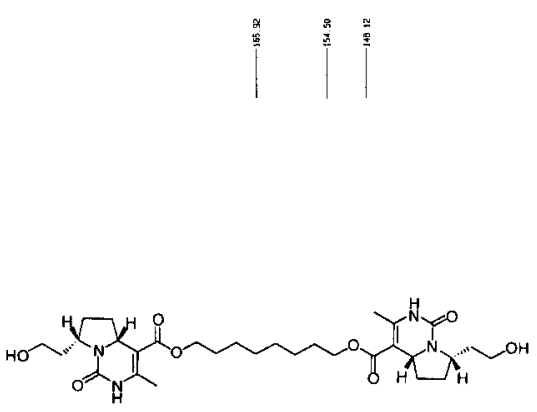

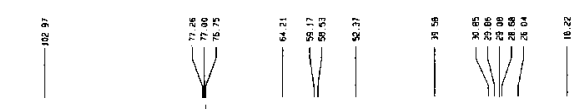

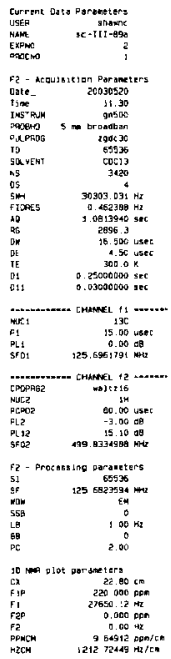




\section{${ }^{1} \mathrm{H}$ and ${ }^{13} \mathrm{C}$ NMR Spectra}

\section{Minor Stereoisomers of Selected Double Biginelli Products}

These samples are typically contaminated with some of the major $C_{2}$-symmetric isomers. 
1H spectrum

s

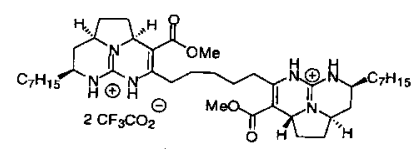

$13 \mathrm{C}$ spectrum with $1 H$ decoupling

$\stackrel{s}{2}$
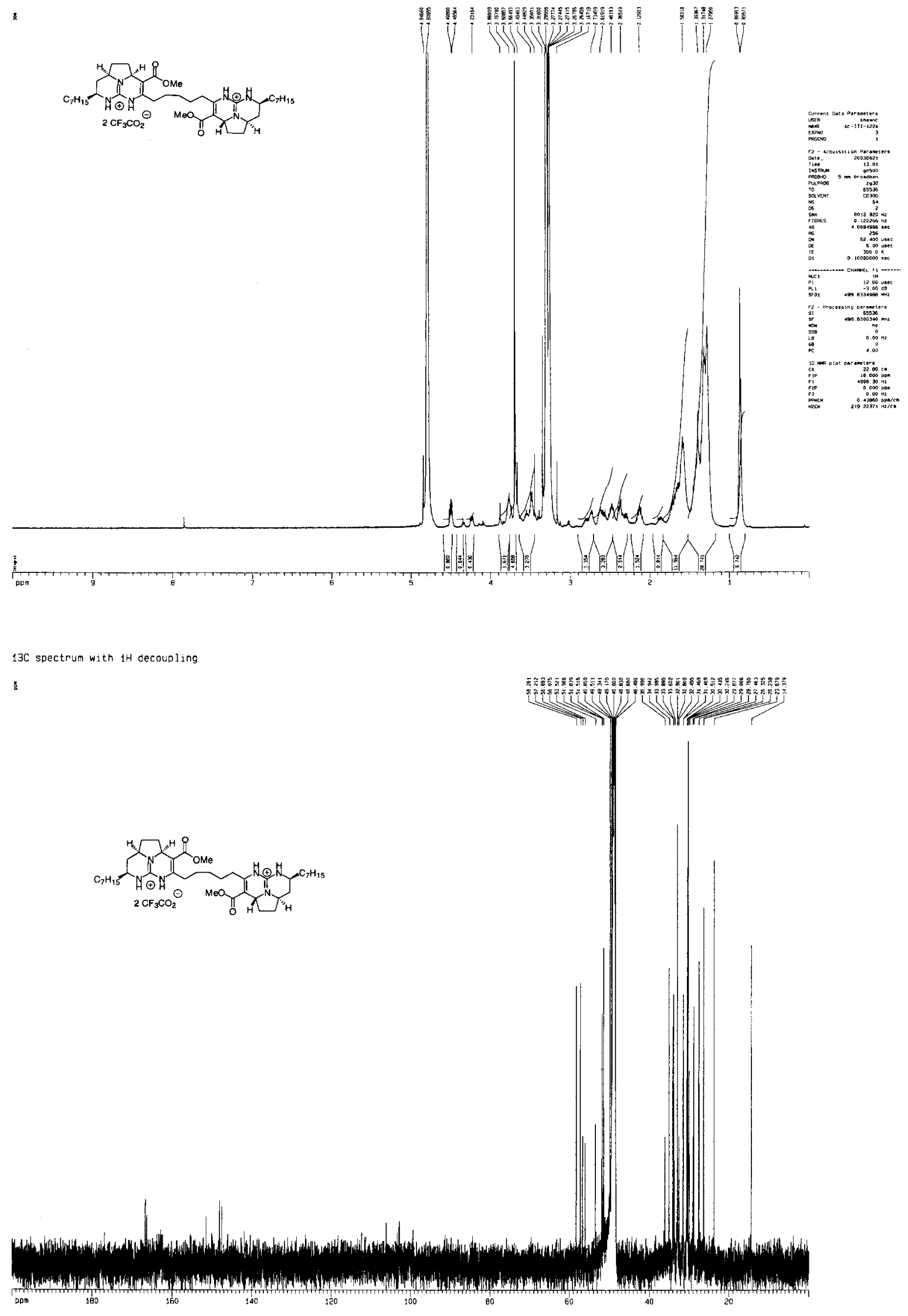

tat

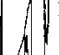

(N)

1.14 II

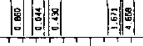
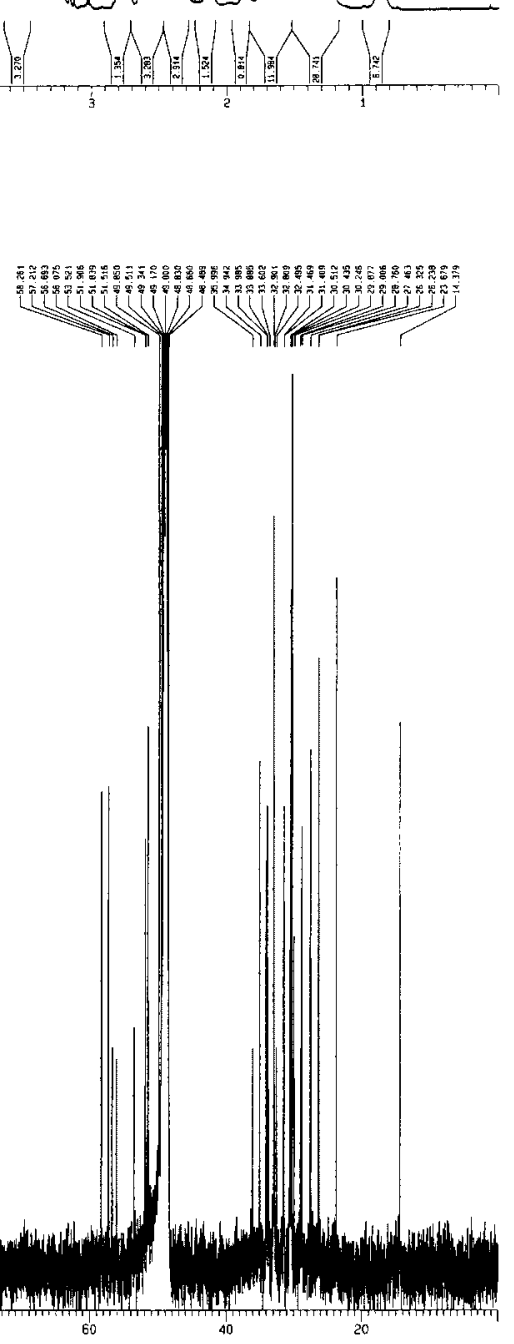
气
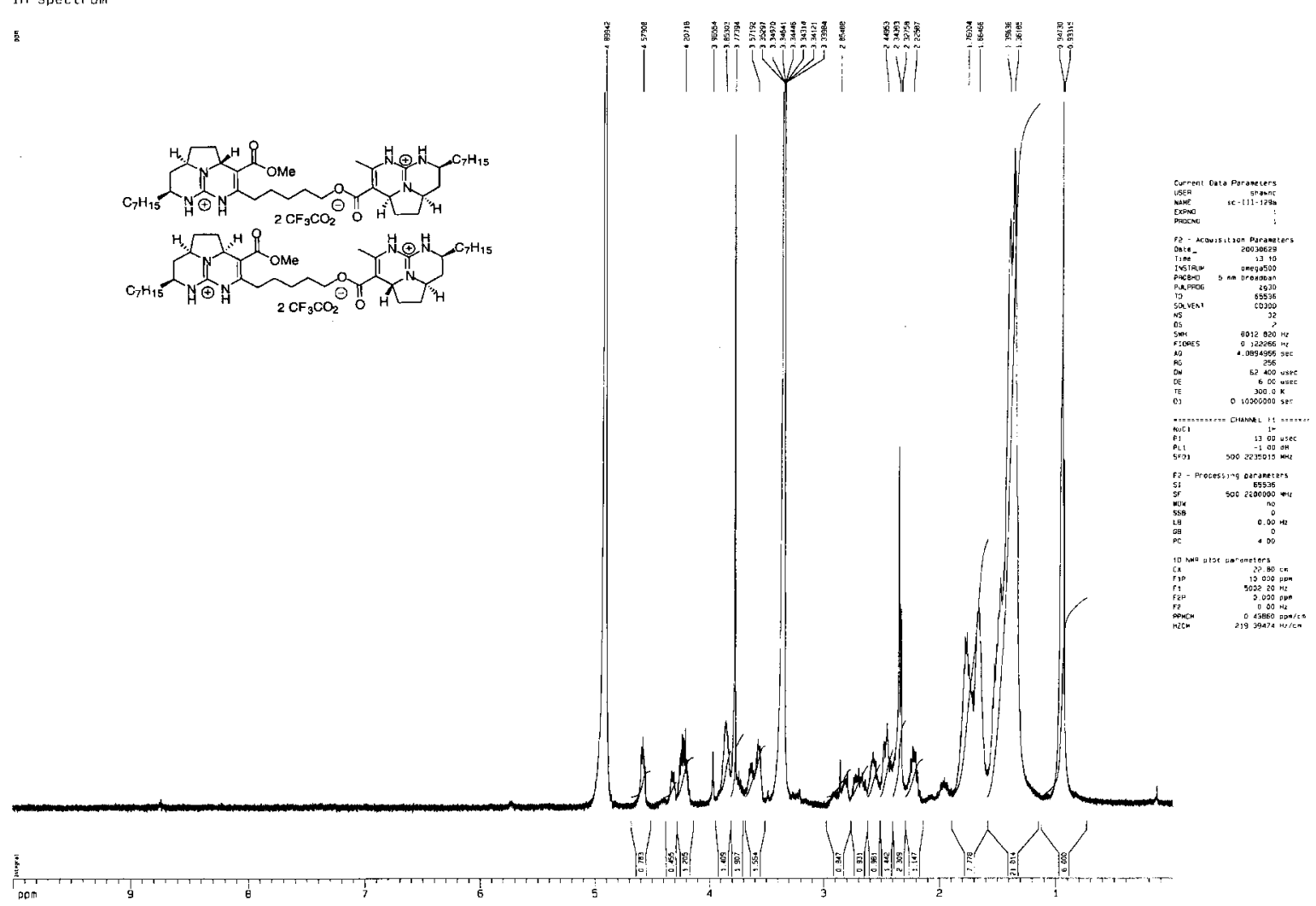

$13 \mathrm{C}$ spectrum with $1 \mathrm{H}$ decoupling

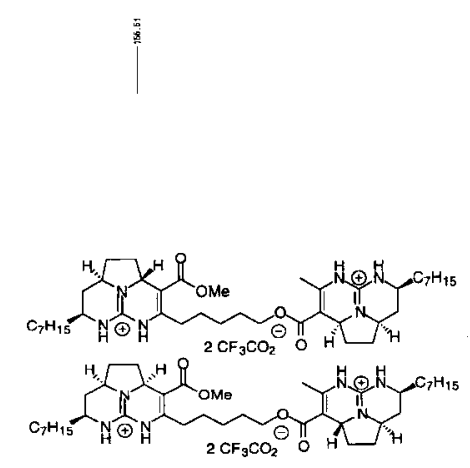

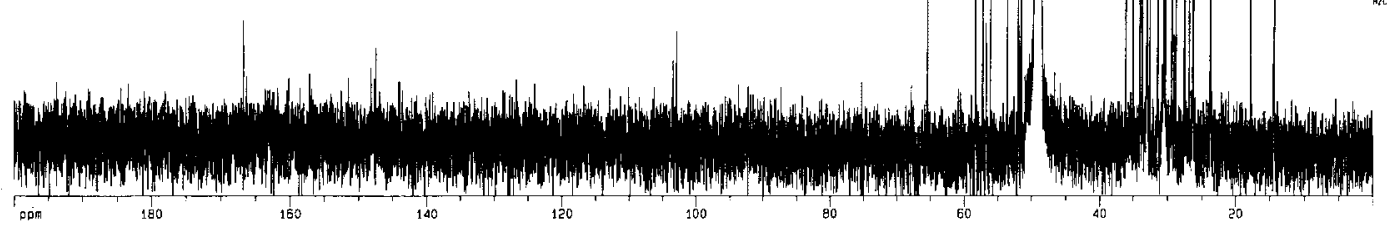

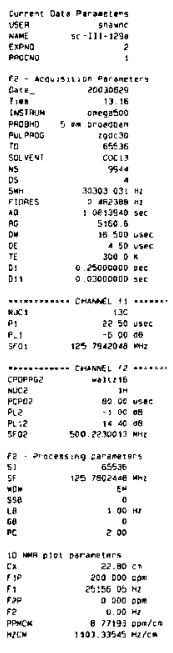




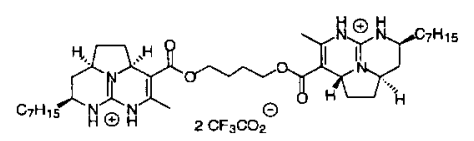

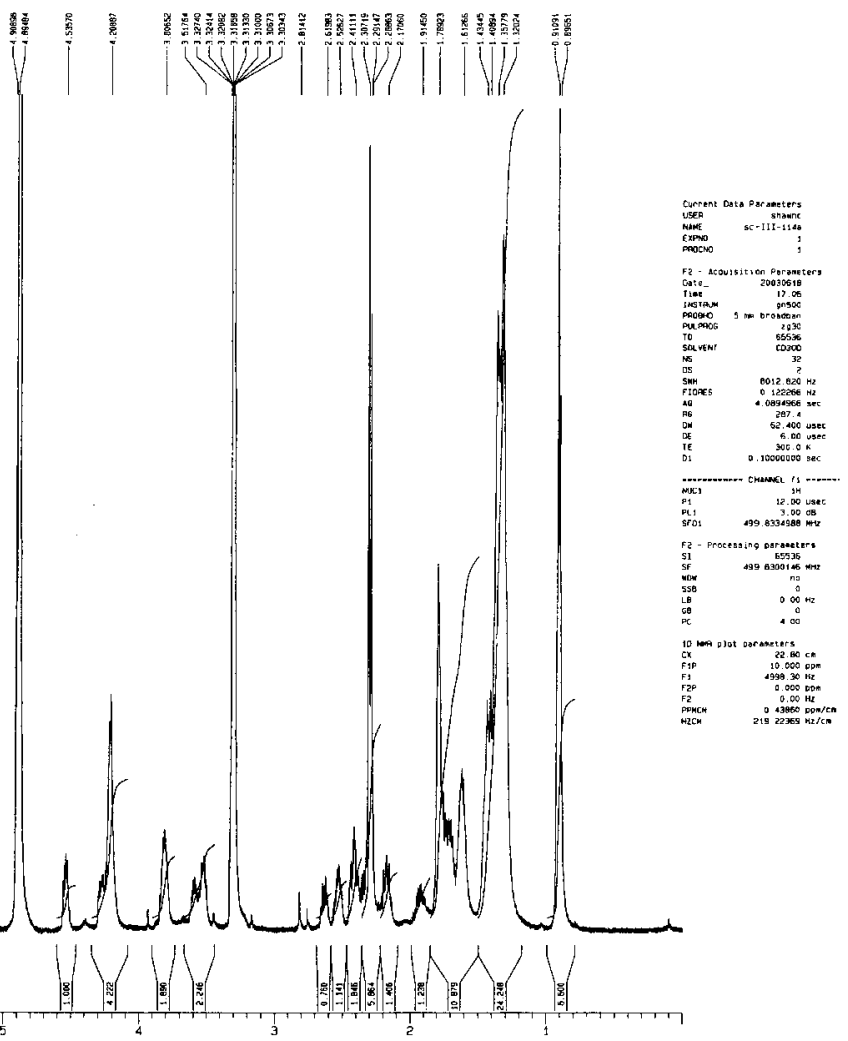

$13 \mathrm{C}$ spectrum with $1 \mathrm{H}$ decoupling

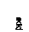

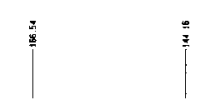

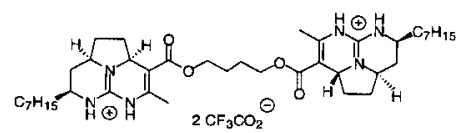

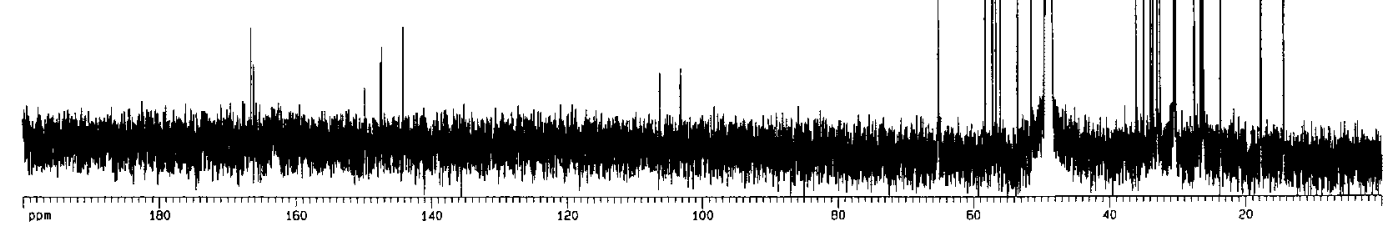




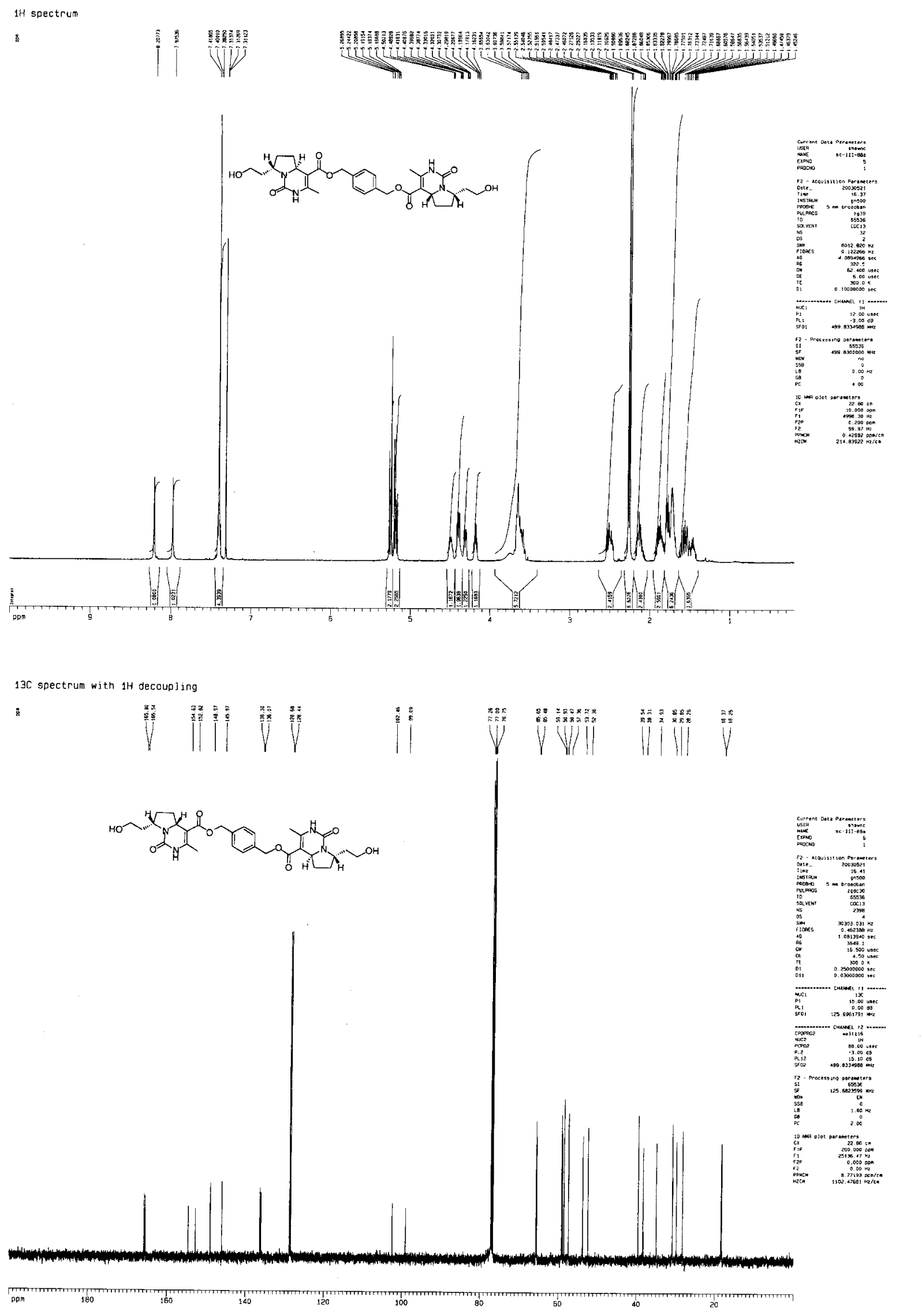




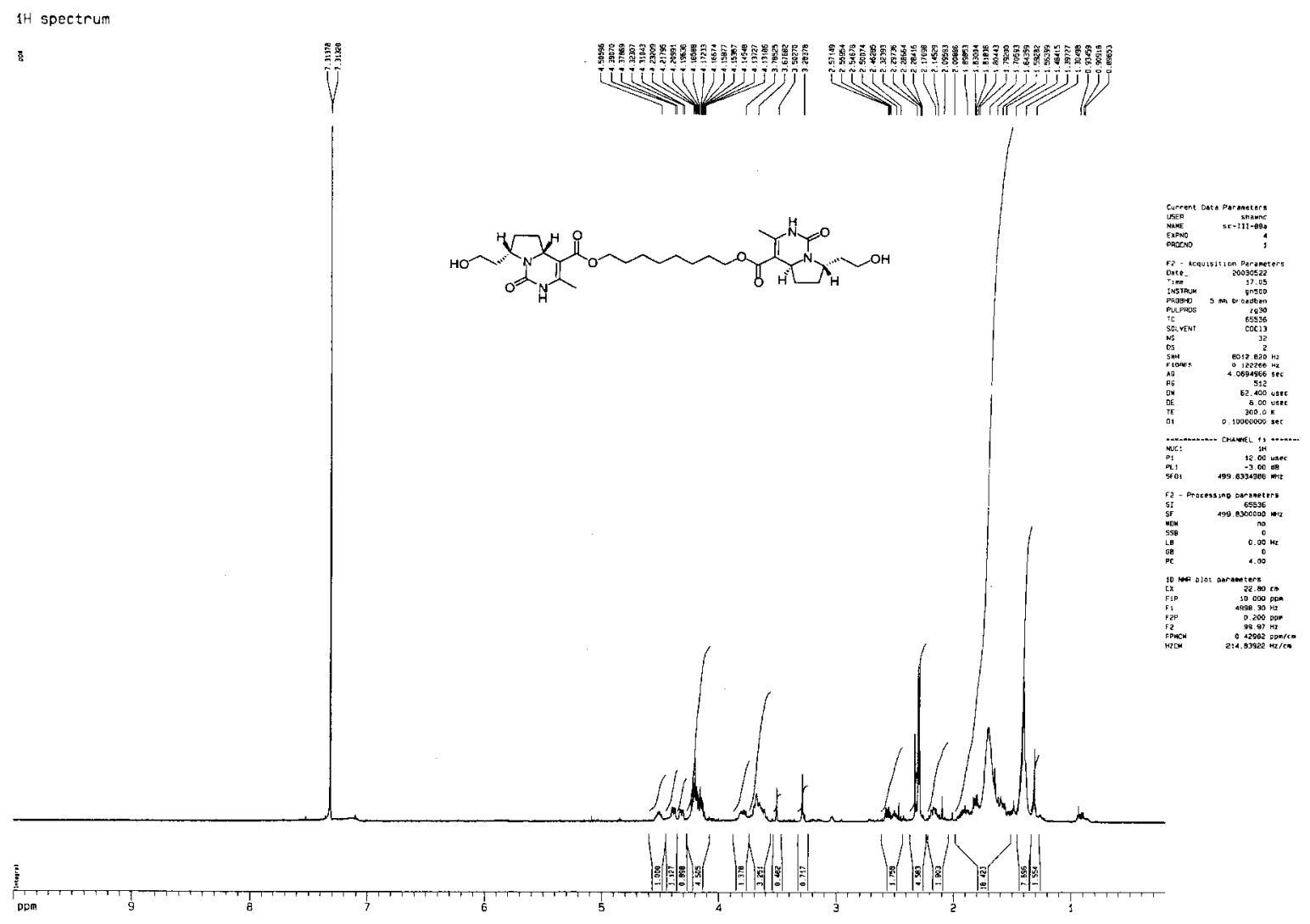

136 spectrum with 1 d decoupling

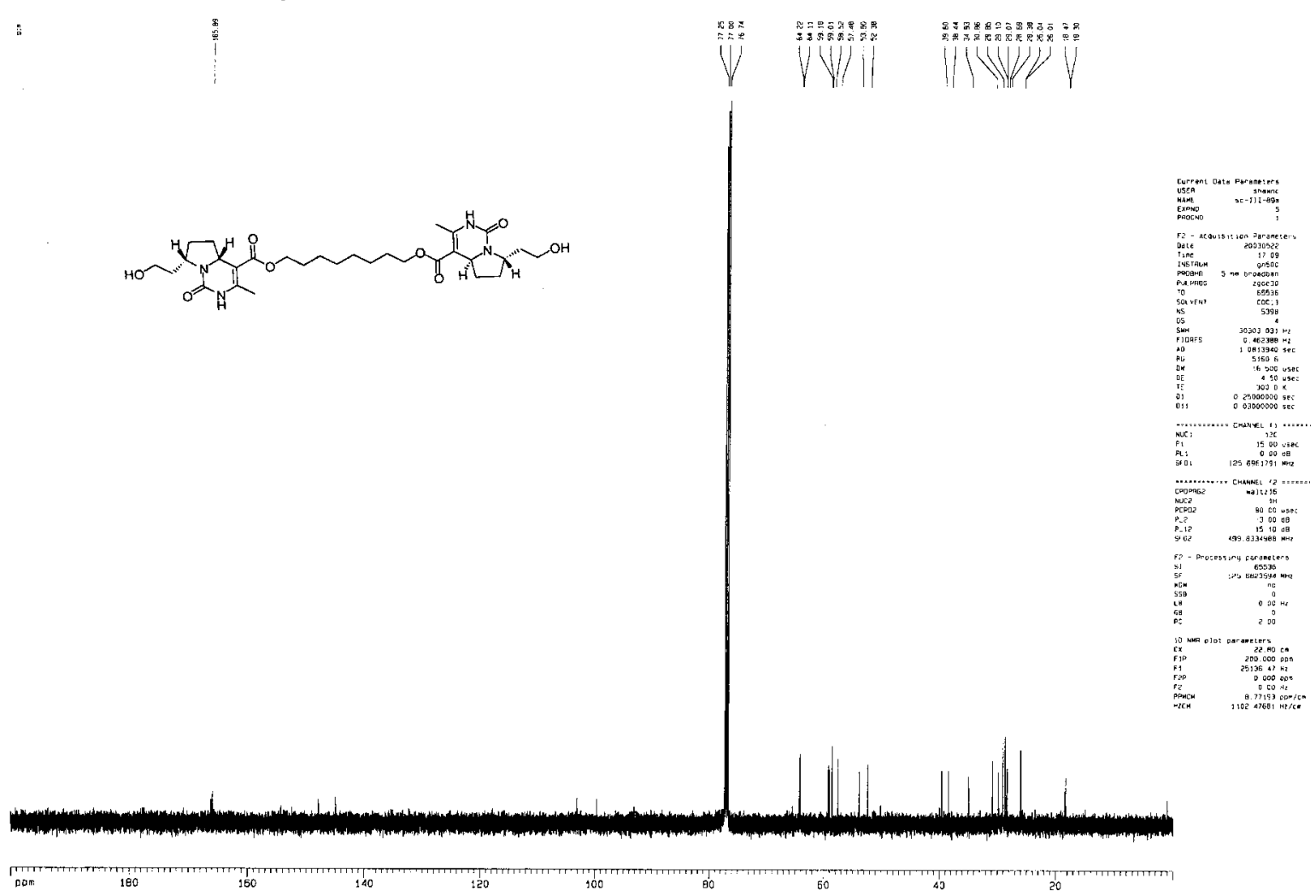




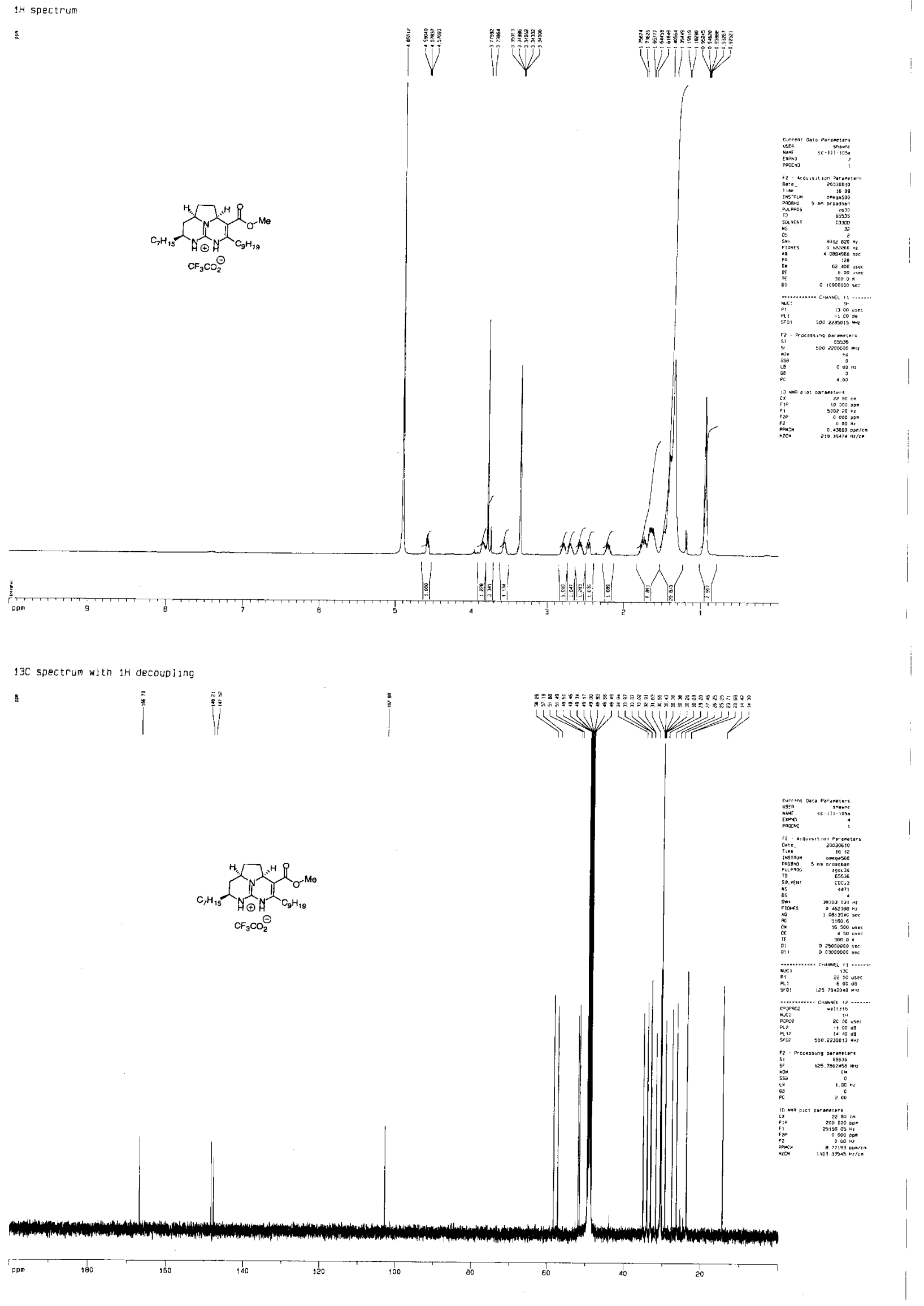

